\title{
ON THE DIMENSION OF SPLINE SPACES ON PLANAR T-MESHES
}

\author{
BERNARD MOURRAIN
}

\begin{abstract}
We analyze the space $\mathcal{S}_{m, m^{\prime}}^{\mathbf{r}}(\mathcal{T})$ of bivariate functions that are piecewise polynomial of bi-degree $\leqslant\left(m, m^{\prime}\right)$ and of smoothness $\mathbf{r}$ along the interior edges of a planar T-mesh $\mathcal{T}$. We give new combinatorial lower and upper bounds for the dimension of this space by exploiting homological techniques. We relate this dimension to the weight of the maximal interior segments of the T-mesh, defined for an ordering of these maximal interior segments. We show that the lower and upper bounds coincide, for high enough degrees or for hierarchical T-meshes which are regular enough. We give a rule of subdivision to construct hierarchical T-meshes for which these lower and upper bounds coincide. Finally, we illustrate these results by analyzing spline spaces of small degrees and smoothness.
\end{abstract}

\section{INTRODUCTION}

Standard parametrisations of surfaces in Computer Aided Geometric Design are based on tensor product B-spline functions, defined from a grid of nodes over a rectangular domain [11. These representations are easy to control but their refinement has some drawback. Inserting a node in one direction of the parameter domain implies the insertion of several control points in the other directions. If, for instance, regions along the diagonal of the parameter domain should be refined, this would create a fine grid in some regions where it is not needed. To avoid this problem, while extending the standard tensor product representation of CAGD, spline functions associated to a subdivision with T-junctions instead of a grid, have been studied. Such a T-mesh is a partition of a domain $\Omega$ into axis-aligned boxes, called the cells of the T-mesh.

The first type of T-splines introduced in 20,21, are defined by blending functions which are products of univariate B-spline basis functions associated to some nodes of the subdivision. They are piecewise polynomial functions, but the pieces where these functions are polynomial do not match with the cells of the T-subdivision. Moreover, there is no proof that these piecewise polynomial functions are linearly independent. Indeed, 3] shows that in some cases, these blending T-spline functions are not linearly independent. Another issue related to this construction is that there is no characterization of the vector space spanned by these functions. For this reason, the partition of unity property which is useful in CAGD is not available directly in this space. The spline functions have to be replaced by piecewise rational functions, so that these piecewise rational functions sum to 1 . However, this construction complexifies the practical use of such T-splines.

Received by the editor May 26, 2013 and, in revised form, December 23, 2011 and July 9, 2012.

2010 Mathematics Subject Classification. Primary 14Q20, 14Q99, 13P25; Secondary 68W30, 65D17, 65D07. 
Being able to describe a basis of the vector space of piecewise polynomials of a given smoothness on a T-mesh is an important but non-trivial issue. It yields a construction of piecewise polynomial functions on the T-subdivision which form a partition of unity so that the use of piecewise rational functions is not required. It has also a direct impact in approximation problems such as surface reconstruction [5] or isogeometric analysis [14, where controlling the space of functions used to approximate a solution is critical. In CAGD, it also provides more degrees of freedom to control a shape. This explains why further works have been developed to understand better the space of piecewise polynomial functions with given smoothness on a T-subdivision.

To tackle these issues special families of splines on T-meshes have been studied. In [6] and [8], these splines are piecewise polynomial functions on a hierarchical T-subdivision. They are called PHT-splines (Polynomial Hierarchical T-splines). Dimension formulae of the spline space on such a subdivision have been proposed when the degree is high enough compared to the smoothness [6], [13, [16] and in some cases for biquadratic $C^{1}$ piecewise polynomial functions [7. The construction of a basis is described for bicubic $C^{1}$ spline spaces in terms of the coefficients of the polynomials in the Bernstein basis attached to a cell. When a cell is subdivided into 4 subcells, the Bernstein coefficients of the basis functions of the old level are modified and new linearly independent functions are introduced, using Bernstein bases on the cells at the new level.

In this paper, we analyse the dimension of the space $\mathcal{S}_{m, m^{\prime}}^{\mathbf{r}}(\mathcal{T})$ of bivariate functions that are piecewise polynomial of bi-degree $\leqslant\left(m, m^{\prime}\right)$ of smoothness $\mathbf{r}$ along the interior edges of a general planar T-mesh $\mathcal{T}$, where $\mathbf{r}$ is a smoothness distribution on $\mathcal{T}$.

As we will see, computing this dimension reduces to compute the dimension of the kernel of a certain linear map (namely the map $\tilde{\partial}_{2}$ introduced in Section 2).

Thus for a given T-mesh, a given smoothness distribution $\mathbf{r}$ and a given bi-degree $\left(m, m^{\prime}\right)$, it is possible to compute the dimension of $\mathcal{S}_{m, m^{\prime}}^{\mathbf{r}}(\mathcal{T})$ by linear algebra tools (see e.g. a software implementation developed by P. Alfed 1 for such computations). We would like to avoid a case-by-case treatment and to describe this dimension in terms of combinatorial quantities attached to $\mathcal{T}$ and easy to evaluate. As shown in [17] or [1, the dimension may also depend on the geometry of the T-mesh and not just on its topology. This explains why it is not always possible to provide a purely combinatorial formula for the dimension of $\mathcal{S}_{m, m^{\prime}}^{\mathbf{r}}(\mathcal{T})$.

The main results in this paper are:

- a description of the dimension in terms of a combinatorial part that depends only on the topology of the T-mesh and an homological part that takes into account the fact that the dimension may depend on the geometry of the T-mesh (Theorem 3.1);

- combinatorial lower and upper bounds on the dimension that are easy to evaluate (Theorem 3.7);

- sufficient conditions under which the lower and upper bounds coincide so that the dimension depends only on the topology of the T-mesh (Theorem [3.9).

\footnotetext{
${ }^{1}$ http://www .math.utah.edu/ pa/MDS/index.html
} 
We proceed as follows. By extending homological techniques developed in 2 . and [19], we obtain combinatorial lower and upper bounds on the dimension of these spline spaces for general T-subdivisions. We relate the upper bound to the maximal interior segments and their weights and show that the lower and upper bounds coincide for $T$-meshes which are regular enough. Namely, if a T-mesh is $\left(m+1, m^{\prime}+1\right)$-weighted, the dimension depends directly on the number of faces, interior edges and interior points. In particular, we obtain the dimension formula for a constant smoothness distribution $\mathbf{r}=\left(r, r^{\prime}\right)$ with $m \geq 2 r+1$ and $m^{\prime} \geq$ $2 r^{\prime}+1$, providing a new proof of a result also available in [6], [13, [16] for a hierarchical T-mesh. The algebraic approach gives an homological interpretation of the method called the Smoothing Cofactor-Conformality method in [23]. It allows us to generalize the dimension formulae obtained by this technique; see [16] and $[13$. We also give a rule of subdivision to construct hierarchical T-meshes for which the lower and upper bounds coincide. As a consequence, we can recover the dimension of the space of Locally Refined splines described in [9]. We do not consider the problem of constructing explicit bases for these spline spaces, which will be analyzed separately.

In the first section, we recall the notation and the polynomial properties which are needed in the following. Section 2 describes the chain complex associated to the spline space and analyzes its homology. In Section 3 , we give lower and upper bounds on the dimension of the spline space and analyze cases where these bounds coincide. Section 4 deals with the properties of hierarchical T-meshes, obtained by recursive subdivisions of cells. In the last section, we analyse some examples for small degree and smoothness.

\section{Planar T-SPlines}

In the following, we will deal with notions which are of topological and algebraic nature. We start by the topological definitions.

1.1. T-meshes. For any set $S \subset \mathbb{R}^{2}, \bar{S}$ is its closure for the usual topology, $S^{\circ}$ its relative interior, $\partial S$ its boundary.

We define a T-mesh $\mathcal{T}$ of $\mathbb{R}^{2}$ as:

- a finite set $\mathcal{T}_{2}$ of closed axis-aligned rectangles of $\mathbb{R}^{2}$,

- a finite set $\mathcal{T}_{1}$ of closed axis-aligned segments included in $\bigcup_{\sigma \in \mathcal{T}_{2}} \partial \sigma$,

- a finite set of points $\mathcal{T}_{0}=\bigcup_{\tau \in \mathcal{T}_{1}} \partial \tau$,

such that:

- For $\sigma \in \mathcal{T}_{2}, \partial \sigma$ is the finite union of elements of $\mathcal{T}_{1}$.

- For $\sigma, \sigma^{\prime} \in \mathcal{T}_{2}$ with $\sigma \neq \sigma^{\prime}, \sigma \cap \sigma^{\prime}=\partial \sigma \cap \partial \sigma^{\prime}$ is the finite union of elements of $\mathcal{T}_{1} \cup \mathcal{T}_{0}$.

- For $\tau, \tau^{\prime} \in \mathcal{T}_{1}$ with $\tau \neq \tau^{\prime}, \tau \cap \tau^{\prime}=\partial \tau \cap \partial \tau^{\prime} \subset \mathcal{T}_{0}$.

We denote by $\Omega=\bigcup_{\sigma \in \mathcal{T}_{2}} \sigma \subset \mathbb{R}^{2}$ and call it the domain of the T-mesh $\mathcal{T}$.

The elements of $\mathcal{T}_{2}$ are called 2-faces or cells and their number is denoted $f_{2}$.

The elements of $\mathcal{T}_{1}$ are called 1 -faces or edges. An element of $\mathcal{T}_{1}$ is called an interior edge if it intersects $\Omega^{\circ}$. It is called a boundary edge otherwise. The set of interior edges is denoted by $\mathcal{T}_{1}^{o}$. The number of edges in $\mathcal{T}_{1}$ is $f_{1}$ and the number of interior edges is $f_{1}^{o}$.

An edge parallel to the first (resp. second) axis of $\mathbb{R}^{2}$ is called horizontal (resp. vertical). Let $\mathcal{T}_{1}^{o, h}$ (resp. $\mathcal{T}_{1}^{o, v}$ ) be the set of horizontal (resp. vertical) interior 
edges and $f_{1}^{h}$ (resp. $f_{1}^{v}$ ) the number of interior horizontal (resp. vertical) edges. Then, the number of interior edges is $f_{1}^{o}=f_{1}^{h}+f_{1}^{v}$.

The elements of $\mathcal{T}_{0}$ are called 0 -faces or vertices. A vertex is interior if it is in $\Omega^{\circ}$. It is a boundary vertex otherwise. The set of interior vertices is denoted $\mathcal{T}_{0}^{o}$. We denote by $f_{0}$ the number of vertices of $\mathcal{T}_{0}$ and by $f_{0}^{o}$ the number of interior vertices.

A vertex is a crossing vertex if it is an interior vertex and belongs to 4 distinct elements of $\mathcal{T}_{1}$. A vertex is a T-vertex if it is an interior vertex and belongs to exactly 3 distinct elements of $\mathcal{T}_{1}$. Let $f_{0}^{+}$(resp. $f_{0}^{T}$ ) be the number of crossing (resp. T) vertices. A boundary vertex is a vertex in $\mathcal{T}_{0} \cap \partial \Omega$. The number of boundary vertices is $f_{0}^{b}$. A vertex is a corner vertex if it belongs to $\partial \Omega$ and to a vertical and a horizontal boundary edge.

To simplify the definitions and remove redundant edges, we will assume that any vertex $\gamma \in \mathcal{T}_{0}$ belongs at least to one horizontal edge $\tau_{h}(\gamma) \in \mathcal{T}_{1}$ and one vertical edge $\tau_{v}(\gamma) \in \mathcal{T}_{1}$.

We denote by $\nu_{h}(\mathcal{T})=\left\{s_{1}, \ldots, s_{l}\right\} \subset \mathbb{R}\left(\right.$ resp. $\left.\nu_{v}(\mathcal{T})=\left\{t_{1}, \ldots, t_{m}\right\} \subset \mathbb{R}\right)$ the set of first (resp. second) coordinates of the points in vertical (horizontal) segments $\in \mathcal{T}_{1}^{v}\left(\right.$ resp. $\left.\in \mathcal{T}_{1}^{h}\right)$. The elements of $\nu_{h}(\mathcal{T})$ (resp. $\left.\nu_{v}(\mathcal{T})\right)$ are called the horizontal (resp. vertical) nodes of the T-mesh $\mathcal{T}$.

Example 1.1. Let us illustrate the previous definitions on the following T-mesh:

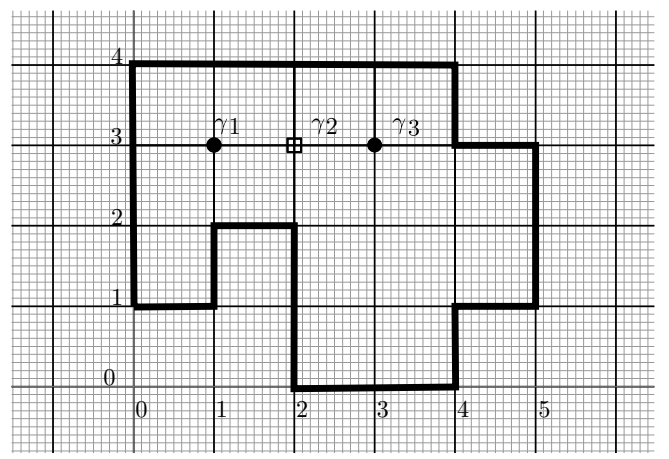

In this example, there are $f_{2}=7$ rectangles, $f_{1}^{o}=9$ interior edges such that $f_{1}^{h}=4$ are horizontal and $f_{1}^{v}=5$ are vertical. There are $f_{0}^{o}=3$ interior points $\gamma_{1}, \gamma_{2}, \gamma_{3}$; $\gamma_{1}, \gamma_{3}$ are T-vertices and $\gamma_{2}$ is a crossing vertex. There are $f_{0}^{b}=15$ boundary vertices and 12 corner vertices.

The horizontal nodes are $\nu_{h}(\mathcal{T})=\{0, \ldots, 5\}$ and the vertical nodes are $\nu_{v}(\mathcal{T})=$ $\{0, \ldots, 4\}$.

For our analysis of spline spaces on T-meshes, we assume the following:

Assumption: The domain $\Omega$ is simply connected and $\Omega^{\circ}$ is connected.

This implies that $\Omega$ has one connected component with no "hole" and that the number of boundary edges through a boundary vertex is 2 .

1.2. T-splines. We are now going to define the spaces of piecewise polynomial functions on a T-mesh, with bounded degrees and given smoothness. An element in such a space is called a spline function.

Definition 1.2. A smoothness distribution on a T-mesh $\mathcal{T}$ is a pair of maps $\left(\mathbf{r}_{h}, \mathbf{r}_{v}\right)$ from $\left(\nu_{h}(\mathcal{T}) \times \nu_{v}(\mathcal{T})\right)$ to $\mathbb{N} \times \mathbb{N}$. 
For convenience, we will define the smoothness map $\mathbf{r}$ on $\mathcal{T}_{1}$ as follows: for any $\tau \in \mathcal{T}_{1}^{v}\left(\right.$ resp. $\left.\tau \in \mathcal{T}_{1}^{h}\right), \mathbf{r}(\tau)=\mathbf{r}_{h}(s)$ (resp. $\mathbf{r}(\tau)=\mathbf{r}_{v}(t)$ ) where $s \in \nu_{h}(\mathcal{T})$ (resp. $t \in \nu_{v}(\mathcal{T})$ ) is the first (resp. second) coordinate of any point of $\tau$. We will also define the horizontal and vertical smoothness on $\mathcal{T}_{0}$ as follows: for any $\gamma=(s, t) \in \mathcal{T}_{0}$, $\mathbf{r}_{h}(\gamma)=\mathbf{r}_{h}(s)$ and $\mathbf{r}_{v}(\gamma)=\mathbf{r}_{v}(t)$.

For $r, r^{\prime} \in \mathbb{N}$, we say that $\mathbf{r}$ is a constant smoothness distribution equal to $\left(r, r^{\prime}\right)$ if $\forall s \in \nu_{h}(\mathcal{T}), \mathbf{r}(s)=r, \forall t \in \nu_{v}(\mathcal{T}), \mathbf{r}(t)=r^{\prime}$

Let $R=\mathbb{R}[s, t]$ be the polynomial ring with coefficients in $\mathbb{R}$. For $m, m^{\prime} \in \mathbb{N}$, we denote by $R_{m, m^{\prime}}=R_{\left(m, m^{\prime}\right)}$ the vector of polynomials in $R$ of degree $\leq m$ in $s$ and $\leq m^{\prime}$ in $t$. An element of $R_{m, m^{\prime}}$ is of bi-degree $\leqslant\left(m, m^{\prime}\right)$.

The goal of this paper is to analyse the dimension of the space of splines of bi-degree $\leq\left(m, m^{\prime}\right)$ and of smoothness $\mathbf{r}$ on a T-mesh $\mathcal{T}$, that we define next.

Definition 1.3. Let $\mathcal{T}$ be a T-mesh and $\mathbf{r}$ a node smoothness distribution. We denote by $\mathcal{S}_{m, m^{\prime}}^{\mathbf{r}}(\mathcal{T})$ the vector space of functions which are polynomials in $R_{m, m^{\prime}}$ on each cell $\sigma \in \mathcal{T}_{2}$ and of class $\mathbf{r}(\tau)$ in $s$ (resp. in $t$ ) at any point of $\tau \cap \Omega^{o}$ if $\tau$ is a vertical (resp. horizontal) interior edge.

We will say that $f \in \mathcal{S}_{m, m^{\prime}}^{\mathbf{r}}(\mathcal{T})$ is of (continuity) class $C^{\mathbf{r}}$ on $\mathcal{T}$. We notice that the boundary edges and their smoothness are not involved in the characterization of a spline function.

Example 1.4. We consider again the T-mesh of Example 1.1. If we take the node smoothness distribution $\mathbf{r}_{h}(1)=1, \mathbf{r}_{h}(2)=0, \mathbf{r}_{h}(3)=\mathbf{r}_{h}(4)=\mathbf{r}_{h}(5)=1$, and $\mathbf{r}_{v}$ constant equal to 1 , then $\mathcal{S}_{3,3}^{\mathbf{r}}(\mathcal{T})$ is the vector space of bicubic piecewise polynomial functions on $\mathcal{T}$ which are $C^{1}$ in $s$ for $s<2$ and $s>2$, continuous for $s=2$ and $C^{1}$ in $t$ in $\Omega^{o}$.

1.3. Polynomial properties. We recall here basic results on the dimension of the vector spaces involved in the analysis of $\mathcal{S}_{m, m^{\prime}}^{\mathbf{r}}(\mathcal{T})$ :

For any $\tau \in \mathcal{T}_{1}$, let $l_{\tau} \in R$ be a non-zero polynomial (of degree 1 ) defining the line supporting the edge $\tau$. Let $\Delta^{\mathbf{r}}(\tau)=l_{\tau}^{\mathbf{r}(\tau)+1}$. We denote by $\mathfrak{I}^{\mathbf{r}}(\tau)=\left(\Delta^{\mathbf{r}}(\tau)\right)$ the ideal generated by the polynomial $\Delta^{\mathbf{r}}(\tau) \in R$ and by $\mathfrak{I}_{m, m^{\prime}}^{\mathbf{r}}(\tau)=\mathfrak{I}^{\mathbf{r}}(\tau) \cap R_{m, m^{\prime}}$ its part of bi-degree $\leq\left(m, m^{\prime}\right)$. Notice that $\mathfrak{I}^{\mathbf{r}}(\tau)$ defines the line supporting the egde $\tau$ with multiplicity $(\mathbf{r}(\tau)+1)$. By definition, two horizontal (resp. vertical) edges $\tau_{1}, \tau_{2}$ which share a point define the same ideal $\mathfrak{I}^{\mathbf{r}}\left(\tau_{1}\right)=\mathfrak{I}^{\mathbf{r}}\left(\tau_{2}\right)$. We define the bi-degree $\delta$ for any edge $\tau \in \mathcal{T}_{1}$ as follows:

- $\delta(\tau)=(\mathbf{r}(\tau)+1,0)$ if $\tau$ is vertical,

- $\delta(\tau)=(0, \mathbf{r}(\tau)+1)$ if $\tau$ is horizontal.

Let $\mathfrak{I}^{\mathbf{r}}(\gamma)=\mathfrak{I}^{\mathbf{r}}\left(\tau_{v}\right)+\mathfrak{I}^{\mathbf{r}}\left(\tau_{h}\right)=\left(\Delta^{\mathbf{r}}\left(\tau_{v}\right), \Delta^{\mathbf{r}}\left(\tau_{h}\right)\right)$ where $\tau_{v}, \tau_{h} \in \mathcal{T}_{1}$ are vertical and horizontal edges such that $\tau_{v} \cap \tau_{h}=\{\gamma\}$. The ideal $\mathfrak{I}^{\mathbf{r}}(\gamma)$ defines the point $\gamma$ with multiplicity $\left(\mathbf{r}_{h}(\gamma)+1\right) \times\left(\mathbf{r}_{v}(\gamma)+1\right)$. We denote by $\mathfrak{I}_{m, m^{\prime}}^{\mathbf{r}}(\gamma)=\mathfrak{I}_{m, m^{\prime}}^{\mathbf{r}}\left(\tau_{v}\right)+$ $\mathfrak{I}_{m, m^{\prime}}^{\mathrm{r}}\left(\tau_{h}\right)$. Notice that these definitions are independent of the choice of the vertical edge $\tau_{v}$ and horizontal edge $\tau_{h}$ which contain $\gamma$. The bi-degree of a vertex $\gamma \in \mathcal{T}_{0}$ is $\delta(\gamma)=\left(\mathbf{r}_{h}(\gamma)+1, \mathbf{r}_{v}(\gamma)+1\right)$.

Here are the basic dimension relations that we will use to analyse the spline functions on a T-mesh.

\section{Lemma 1.5.}

$$
\text { - } \operatorname{dim} R_{m, m^{\prime}}=(m+1) \times\left(m^{\prime}+1\right) .
$$


- $\operatorname{dim}\left(R_{m, m^{\prime}} / \mathfrak{I}_{m, m^{\prime}}^{\mathbf{r}}(\tau)\right)= \begin{cases}(m+1) \times\left(\min \left(\mathbf{r}(\tau), m^{\prime}\right)+1\right) & \text { if } \tau \in \mathcal{T}_{1}^{h}, \\ (\min (\mathbf{r}(\tau), m)+1) \times\left(m^{\prime}+1\right) & \text { if } \tau \in \mathcal{T}_{1}^{v} ;\end{cases}$

- $\operatorname{dim}\left(R_{m, m^{\prime}} / \mathfrak{I}_{m, m^{\prime}}^{\mathbf{r}}(\gamma)\right)=\left(\min \left(\mathbf{r}_{h}(\gamma), m\right)+1\right) \times\left(\min \left(\mathbf{r}_{v}(\gamma), m^{\prime}\right)+1\right)$ for all $\gamma \in \mathcal{T}_{0}$.

Proof. To obtain these formulae, we directly check that

- a basis of $R_{m, m^{\prime}}$ is the set of monomials $s^{i} t^{j}$ with $0 \leq i \leq m, 0 \leq j \leq m^{\prime}$;

- a basis of $R_{m, m^{\prime}} / \mathfrak{I}_{m, m^{\prime}}^{\mathrm{r}}(\tau)$ is the set of monomials $s^{i} t^{j}$ with $0 \leq i \leq m$ and $0 \leq j \leq \min \left(\mathbf{r}(\tau), m^{\prime}\right)$ if $\tau \in \mathcal{T}_{1}^{h}$ (resp. $0 \leq i \leq \min (\mathbf{r}(\tau), m), 0 \leq j \leq m^{\prime}$ if $\left.\tau \in \mathcal{T}_{1}^{v}\right)$

- a basis of $R_{m, m^{\prime}} / \mathfrak{I}_{m, m^{\prime}}^{\mathbf{r}}(\gamma)$ is the set of monomials $s^{i} t^{j}$ with $0 \leq i \leq$ $\min \left(\mathbf{r}_{h}(\gamma), m\right), 0 \leq j \leq \min \left(\mathbf{r}_{v}(\gamma), m^{\prime}\right)$.

since the ideal of an edge $\tau \in \mathcal{T}_{1}$ is up to a translation $\left(s^{\mathbf{r}(\tau)+1}\right)$ or $\left(t^{\mathbf{r}(\tau)+1}\right)$.

An algebraic way to characterize the $C^{\mathbf{r}}$-smoothness is given by the next lemma:

Lemma $1.6([2])$. Let $\tau \in \mathcal{T}_{1}$ and let $p_{1}, p_{2}$ be two polynomials. Their derivatives coincide on $\tau$ up to order $\mathbf{r}(\tau)$ iff $p_{1}-p_{2} \in \mathfrak{I}^{\mathbf{r}}(\tau)$.

In the following, we will need algebraic properties on univariate polynomials. We denote by $U=\mathbb{R}[u]$ the space of univariate polynomials in the variable $u$ with coefficients in $\mathbb{R}$. Let $U_{n}$ denote the space of polynomials of $U$ of degree $\leq n$. For a polynomial $g \in U$ of degree $d$ and an integer $n \geq d, g U_{n-d}$ is the vector space of multiples of $g$ which are of degree $\leq n$. For polynomials $g_{1}, \ldots, g_{k} \in U$ respectively of degree $d_{1}, \ldots, d_{k}$ and an integer $n \geq \max _{i=1, \ldots, k} d_{i}, \sum_{i=1}^{k} g_{i} U_{n-d_{i}}$ is the vector space of sums of multiples of $g_{i}$ of degree $\leq n$.

We will use the apolar product defined on $U_{n}$ by

$$
\langle f, g\rangle_{n}=\sum_{i=0}^{n}\left(\begin{array}{c}
n \\
i
\end{array}\right) f_{i} g_{i}
$$

where $f=\sum_{i=0}^{n} f_{i} u^{i}, g=\sum_{i=0}^{n} g_{i} u^{i} \in \mathbb{R}[u]_{n}$. One of the properties that we will need is the following [18, [15], [10]:

Lemma 1.7. Let $g \in U_{n}, d<n$ and $a \in \mathbb{R}$. Then $g$ is orthogonal to $(u-a)^{d} U_{n-d}$ for the apolar product iff

$$
\partial^{k} g(a)=0, k=0, \ldots, n-d .
$$

Proposition 1.8. Let $a_{1}, \ldots, a_{l} \in \mathbb{R}$ be $l$ distinct points and $d_{1}, \ldots, d_{l} \in \mathbb{N}$. Then

$$
\operatorname{dim}\left(\sum_{i=1}^{l}\left(u-a_{i}\right)^{d_{i}} U_{n-d_{i}}\right)=\min \left(n+1, \sum_{i=1}^{l} n-d_{i}+1\right) .
$$

Proof. In order to compute the dimension of $V:=\sum_{i=1}^{l}\left(u-a_{i}\right)^{d} U_{n-d} \subset U_{n}$, we compute the dimension of the orthogonal $V^{\perp}$ in $U_{n}$ of $V$ for the apolar product. Let $g \in U_{n}$ be an element of the orthogonal $V^{\perp}$ of $V$. By Lemma 1.7, $\partial^{k} g\left(a_{i}\right)=$ $0, k=0, \ldots, n-d_{i}, i=1, \ldots, l$. In other words, $g$ is divisible by $\left(u-a_{i}\right)^{n-d_{i}+1}$ for $i=1, \ldots, l$. As the points $a_{i}$ are distinct, $g$ is divisible by

$$
\Pi:=\prod_{i=1}^{l}\left(u-a_{i}\right)^{n-d_{i}+1} .
$$

Conversely, any multiple of $\Pi$ of degree $\leqslant n$ is in $V^{\perp}$. Thus $V^{\perp}=(\Pi) \cap U_{n}$. 
This vector space $V^{\perp}$ of multiples of $\Pi$ in degree $n$ is of dimension $\max (0, n+$ $1-\operatorname{deg}(\Pi))$, so $V$ is of dimension

$$
\begin{aligned}
n+1-\max (0, n+1-\operatorname{deg}(\Pi)) & =\min (n+1, \operatorname{deg}(\Pi)) \\
& =\min \left(n+1, \sum_{i=1}^{l} n-d_{i}+1\right) .
\end{aligned}
$$

We are going to use an equivalent formulation of this result:

$$
\operatorname{dim}\left(U_{n} / \sum_{i=1}^{l}\left(u-a_{i}\right)_{i}^{d} U_{n-d_{i}}\right)=\left(n+1-\sum_{i=1}^{l} n-d_{i}+1\right)_{+},
$$

where for any $a \in \mathbb{Z}, a_{+}=\max (0, a)$. A similar result is proved in 16, Lemma 2 ] when all $d_{i}$ are equal to $d$, by analyzing the coefficient matrix of generators of $\sum_{i=1}^{l}\left(u-a_{i}\right)^{d} U_{n-d}$.

1.4. Maximal interior segments. In order to simplify the analysis of $\mathcal{S}_{m, m^{\prime}}^{\mathbf{r}}(\mathcal{T})$, we introduce the following definitions:

For any interior edge $\tau \in \mathcal{T}_{1}^{o}$, we define $\rho(\tau)$ as the maximal segment made of edges $\in \mathcal{T}_{1}^{o}$ of the same direction as $\tau$, which contains $\tau$ and such that their union is connected. We say that the maximal segment $\rho(\tau)$ is interior if it does not intersect the boundary of $\Omega$.

As all the edges belonging to a maximal segment $\rho$ have the same supporting line, we can define $\Delta^{\mathbf{r}}(\rho)=\Delta^{\mathbf{r}}(\tau)$ for any edge $\tau$ belonging to $\rho=\rho(\tau)$.

The set of all maximal interior segments is denoted by $\operatorname{Mis}(\mathcal{T})$. The set of horizontal (resp. vertical) maximal interior segments of $\mathcal{T}$ is denoted by $\operatorname{MIS}_{h}(\mathcal{T})$ $\left(\right.$ resp. $\left.\operatorname{MIS}_{v}(\mathcal{T})\right)$.

The degree of $\rho \in \operatorname{MIS}(\mathcal{T})$ is by definition $\delta(\rho)=\delta(\tau)$ for any $\tau \subset \rho$.

For each interior vertex $\gamma \in \mathcal{T}_{0}^{o}$, which is the intersection of a horizontal edge $\tau_{h} \in \mathcal{T}_{1}^{o}$ with a vertical edge $\tau_{v} \in \mathcal{T}_{1}^{o}$, let $\rho_{h}(\gamma)$ (resp. $\left.\rho_{v}(\gamma)\right)$ is the corresponding horizontal (resp. vertical) maximal segment. We denote by $\Delta_{h}^{\mathbf{r}}(\gamma)\left(\operatorname{resp} . \Delta_{v}^{\mathbf{r}}(\gamma)\right)$ the equations of the corresponding supporting lines to the power $\mathbf{r}_{h}(\gamma)+1$ (resp. $\left.\mathbf{r}_{v}(\gamma)+1\right)$

Notice that the intersection of two distinct maximal interior segments is either a T-vertex or a crossing vertex.

We say that $\rho \in \operatorname{Mis}(\mathcal{T})$ is blocking $\rho^{\prime} \in \operatorname{MIS}(\mathcal{T})$ if one of the end points of $\rho^{\prime}$ is in the interior of $\rho$.

Example 1.9. In the figure below, the maximal interior edges are indicated by plain segments. 


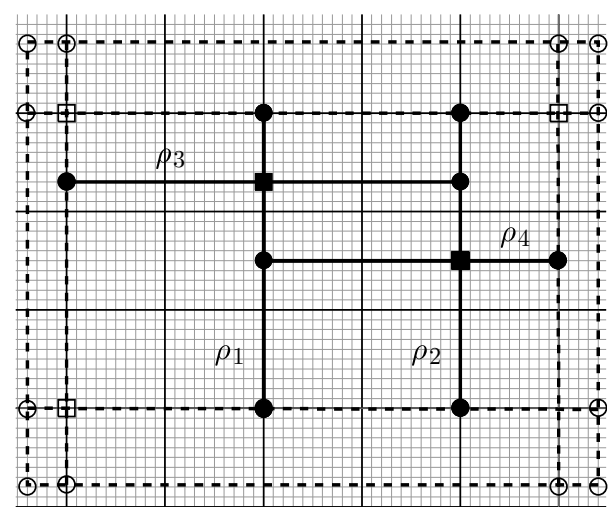

In this example, $\rho_{1}$ is blocking $\rho_{4}$ and $\rho_{2}$ is blocking $\rho_{3}$.

\section{Topological Chain COMPleXes}

In this section, we describe the tools from algebraic topology, that we will use. For more details, see e.g. [22] and [12].

2.1. Definitions. We consider the following complexes:

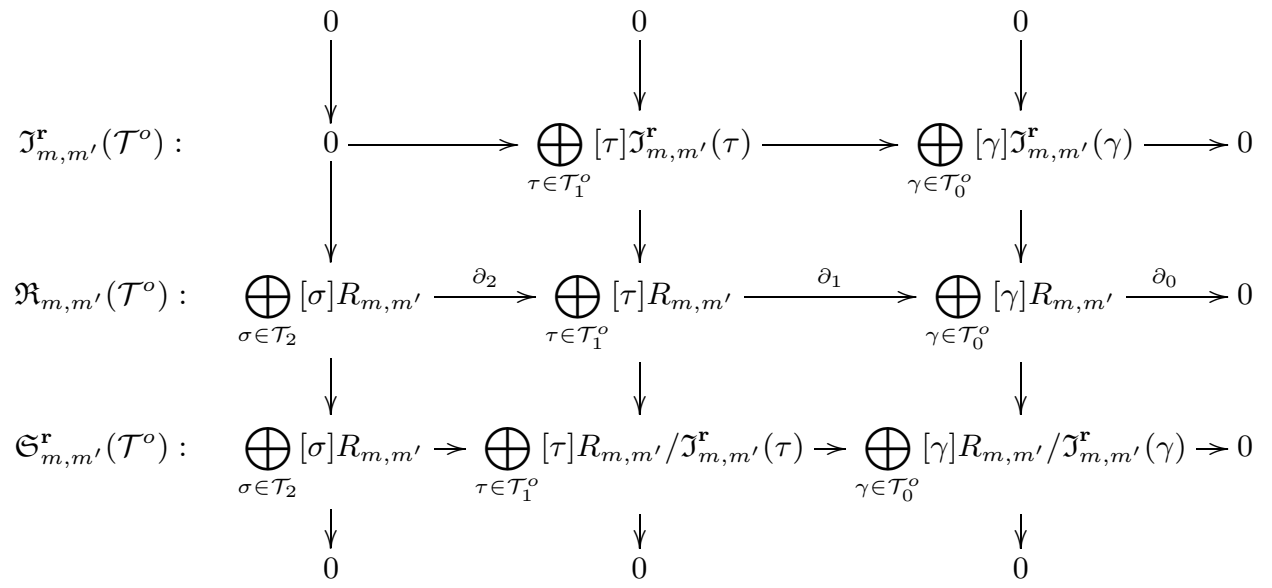

The different vector spaces of these complexes are obtained as the components in bi-degree $\leqslant\left(m, m^{\prime}\right)$ of $R$-modules generated by (formal) independent elements $[\sigma],[\tau],[\gamma]$ indexed respectively by the faces, the interior edges and interior points of $\mathcal{T}$. An oriented edge $\tau \in \mathcal{T}_{1}$ is represented as: $[\tau]=[a b]$ where $a, b \in \mathcal{T}_{0}$ are the end points. The opposite edge is represented by $[b a]$. By convention, $[b a]=-[a b]$.

The maps of the complex $\mathfrak{R}_{m, m^{\prime}}\left(\mathcal{T}^{o}\right)$ are defined as follows:

- for each face $\sigma \in \mathcal{T}_{2}$ with its counterclockwise boundary formed by edges $\tau_{1}=a_{1} a_{2}, \ldots, \tau_{l}=a_{l} a_{1}, \partial_{2}(\sigma)=\left[\tau_{1}\right] \oplus \cdots \oplus\left[\tau_{l}\right]=\left[a_{1} a_{2}\right] \oplus \cdots \oplus\left[a_{l} a_{1}\right] ;$

- for each interior edge $\tau \in \mathcal{T}_{1}^{o}$ from $\gamma_{1}$ to $\gamma_{2} \in \mathcal{T}_{0}, \partial_{1}([\tau])=\left[\gamma_{2}\right]-\left[\gamma_{1}\right]$ where $[\gamma]=0$ if $\gamma \notin \mathcal{T}_{0}^{o}$;

- for each interior point $\gamma \in \mathcal{T}_{0}^{o}, \partial_{0}([\gamma])=0$.

By construction, we have $\partial_{i} \circ \partial_{i+1}=0$ for $i=0,1$. The maps of the complex $\mathfrak{I}_{m, m^{\prime}}^{\mathbf{r}}\left(\mathcal{T}^{o}\right)$ are obtained from those of $\mathfrak{R}_{m, m^{\prime}}\left(\mathcal{T}^{o}\right)$ by restriction, those of the complex $\mathfrak{S}_{m, m^{\prime}}^{\mathbf{r}}\left(\mathcal{T}^{o}\right)$ are obtained by taking the quotient by the corresponding vector 
spaces of $\mathfrak{I}_{m, m^{\prime}}^{\mathrm{r}}\left(\mathcal{T}^{o}\right)$. The corresponding differentials of the complex are denoted $\bar{\partial}_{i}$.

For each column of the diagram, the vertical maps are respectively the inclusion map and the quotient map.

The complex $\Re_{m, m^{\prime}}\left(\mathcal{T}^{o}\right)$ is also known as the chain complex of $\mathcal{T}$ relative to its boundary $\partial \mathcal{T}$.

Example 2.1. We consider the following subdivision $\mathcal{T}$ of a rectangle $\Omega$ :

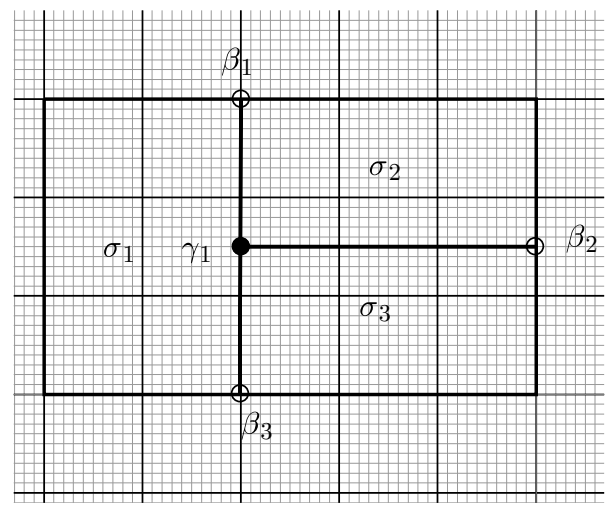

We have

- $\partial_{2}\left(\left[\sigma_{1}\right]\right)=\left[\gamma_{1} \beta_{1}\right]+\left[\beta_{3} \gamma_{1}\right], \partial_{2}\left(\left[\sigma_{2}\right]\right)=\left[\gamma_{1} \beta_{2}\right]+\left[\beta_{1} \gamma_{1}\right], \partial_{2}\left(\left[\sigma_{3}\right]\right)=\left[\gamma_{1} \beta_{3}\right]+$ $\left[\beta_{2} \gamma_{1}\right]$

- $\partial_{1}\left(\left[\beta_{1} \gamma_{1}\right]\right)=\left[\gamma_{1}\right], \partial_{1}\left(\left[\beta_{2} \gamma_{1}\right]\right)=\left[\gamma_{1}\right], \partial_{1}\left(\left[\beta_{3} \gamma_{1}\right]=\left[\gamma_{1}\right]\right.$,

- $\partial_{0}\left(\left[\gamma_{1}\right]\right)=0$.

This defines the following complex:

$$
\mathfrak{R}_{m, m^{\prime}}(\mathcal{T}): \quad \bigoplus_{i=1}^{3}\left[\sigma_{i}\right] R_{m, m^{\prime}} \rightarrow \bigoplus_{i=1}^{3}\left[\beta_{i} \gamma_{1}\right] R_{m, m^{\prime}} \rightarrow\left[\gamma_{1}\right] R_{m, m^{\prime}} \rightarrow 0 .
$$

The matrices of these maps in the canonical (monomial) bases are

$$
\left[\partial_{2}\right]=\left(\begin{array}{ccc}
-I & I & 0 \\
0 & -I & I \\
I & 0 & -I
\end{array}\right),\left[\partial_{1}\right]=\left(\begin{array}{lll}
I & I & I
\end{array}\right),
$$

where $I$ is the identity matrix of size $(m+1) \times\left(m^{\prime}+1\right)$ (i.e., the dimension of $R_{m, m^{\prime}}$.

Let us consider the case where $\gamma_{1}=(0,0),\left(m, m^{\prime}\right)=(2,2)$ and $\mathbf{r}$ is the constant distribution $(1,1)$ on $\mathcal{T}$. The matrices of the complex $\mathfrak{S}_{2,2}^{1,1}(\mathcal{T})$ are

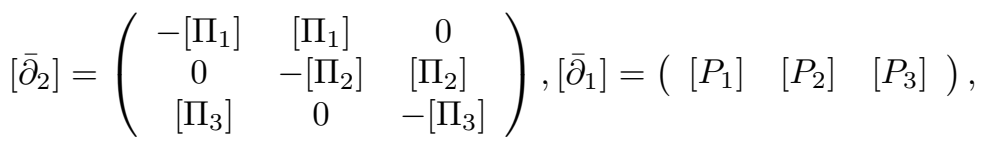

where $\left[\Pi_{i}\right]$ (resp. $\left.\left[P_{i}\right]\right)$ are the matrices of the projections

$$
\begin{array}{rlrl}
\Pi_{1}=\Pi_{3}: R_{2,2} & \rightarrow R_{2,2} /\left(s^{2}\right) & \Pi_{2}: R_{2,2} & \rightarrow R_{2,2} /\left(t^{2}\right) \\
p & \mapsto p \bmod s^{2} & p & \mapsto p \bmod t^{2} \\
& & & \\
P_{1}=P_{3}: R_{2,2} /\left(s^{2}\right) & \rightarrow R_{2,2} /\left(s^{2}, t^{2}\right) & P_{2}: R_{2,2} /\left(t^{2}\right) & \rightarrow R_{2,2} /\left(s^{2}, t^{2}\right) \\
p \bmod s^{2} & \mapsto p \bmod \left(s^{2}, t^{2}\right) & p \bmod t^{2} & \mapsto p \bmod \left(s^{2}, t^{2}\right) .
\end{array}
$$

The matrices $\Pi_{i}$ are of size $12 \times 16$ and the matrices $P_{i}$ are of size $9 \times 12$. 
2.2. Their homology. In this section, we analyse the homology of the different complexes. The homology of a chain complex of a triangulation of a (planar) domain is well known [22, Chap. 4], [12, Chap. 2]. Since, it is not explicit in the literature, we give in the appendix a simple proof of the exactness of $\mathfrak{R}_{m, m^{\prime}}\left(\mathcal{T}^{o}\right)$.

2.2.1. The 0-homology. We start by analysing the homology on the vertices.

Lemma 2.2. $H_{0}\left(\Re_{m, m^{\prime}}\left(\mathcal{T}^{o}\right)\right)=H_{0}\left(\mathfrak{S}_{m, m^{\prime}}^{\mathbf{r}}\left(\mathcal{T}^{o}\right)\right)=0$.

Proof. By Proposition D.1 in the appendix, we have $H_{0}\left(\Re_{m, m^{\prime}}\left(\mathcal{T}^{o}\right)\right)=0$. Taking the quotient by $\mathfrak{I}_{m, m^{\prime}}^{\mathbf{r}}\left(\tau_{i}\right)$, we still get that $\bar{\partial}_{1}$ is surjective so that $H_{0}\left(\mathfrak{S}_{m, m^{\prime}}^{\mathbf{r}}\left(\mathcal{T}^{o}\right)\right)=$ 0 .

We describe the following in more detail:

$$
H_{0}\left(\mathfrak{I}_{m, m^{\prime}}^{\mathbf{r}}\left(\mathcal{T}^{o}\right)\right)=\bigoplus_{\gamma \in \mathcal{T}_{0}^{o}}[\gamma] \mathfrak{I}^{\mathbf{r}}(\gamma) / \partial_{1}\left(\bigoplus_{\tau \in \mathcal{T}_{1}^{o}}[\tau] \mathfrak{I}^{\mathbf{r}}(\tau)\right)
$$

We consider the free $R$-module generated by the half-edge elements $[\gamma \mid \tau]$, for all interior edges $\tau \in \mathcal{T}_{1}^{o}$ and all vertices $\gamma \in \tau$. By convention $[\gamma \mid \tau] \equiv 0$ if $\gamma \in \partial \Omega$.

For $\gamma \in \mathcal{T}_{0}^{o}$, let $E_{h}(\gamma)$ (resp. $E_{v}(\gamma)$ ) be the set of horizontal (resp. vertical) interior edges that contain $\gamma$ and let $E(\gamma)=E_{h}(\gamma) \cup E_{v}(\gamma)$. We consider first the map

$$
\begin{aligned}
\varphi_{\gamma}: \bigoplus_{\tau \in E(\gamma)}[\gamma \mid \tau] R_{\left(m, m^{\prime}\right)-\delta(\tau)} & \rightarrow[\gamma] \mathfrak{I}_{m, m^{\prime}}^{\mathbf{r}}(\gamma), \\
{[\gamma \mid \tau] } & \mapsto[\gamma] \Delta^{\mathbf{r}}(\tau) .
\end{aligned}
$$

By definition of $\mathfrak{I}_{m, m^{\prime}}^{\mathbf{r}}(\gamma)$, this map is surjective. Its kernel is denoted $\mathfrak{K}_{m, m^{\prime}}^{\mathbf{r}}(\gamma)$. Let $P_{h}(\gamma)$ (resp. $P_{v}(\gamma)$ ) be the set of pairs $\left(\tau, \tau^{\prime}\right)$ of distinct horizontal (resp. vertical) interior edges which contain $\gamma$ (with $\left(\tau, \tau^{\prime}\right)$ identify to $\left.\left(\tau^{\prime}, \tau\right)\right)$. We denote by $P(\gamma)=P_{h}(\gamma) \cup P_{v}(\gamma)$. If $\gamma$ is a T-junction, one of the two sets is empty and the other is a singleton containing one pair. If $\gamma$ is a crossing vertex, each set is a singleton.

The following proposition gives an explicit description of the kernel $\mathfrak{K}_{m, m^{\prime}}^{\mathbf{r}}(\gamma)$, that we will exploit hereafter.

\section{Proposition 2.3.}

$$
\begin{aligned}
\mathfrak{K}_{m, m^{\prime}}^{\mathbf{r}}(\gamma) & =\sum_{\left(\tau, \tau^{\prime}\right) \in P(\gamma)}\left([\gamma \mid \tau]-\left[\gamma \mid \tau^{\prime}\right]\right) R_{\left(m, m^{\prime}\right)-\delta(\tau)} \\
& +\sum_{\tau \in E_{h}(\gamma), \tau^{\prime} \in E_{v}(\gamma)}\left([\gamma \mid \tau] \Delta^{\mathbf{r}}\left(\tau^{\prime}\right)-\left[\gamma \mid \tau^{\prime}\right] \Delta^{\mathbf{r}}(\tau)\right) R_{\left(m-r-1, m^{\prime}-r^{\prime}-1\right)}
\end{aligned}
$$

Proof. Let us suppose first that $\gamma$ is a crossing vertex. We denote by $\tau_{1}, \tau_{2}$ the horizontal edges, $\tau_{3}, \tau_{4}$ the vertical edges at $\gamma$. The matrix of the map $\varphi_{\gamma}$ in the basis $\left[\gamma \mid \tau_{i}\right]$ is

$$
\left[\varphi_{\gamma}\right]=\left(\begin{array}{cccc}
\Delta & \Delta & \Delta^{\prime} & \Delta^{\prime}
\end{array}\right)
$$

where $\Delta=\Delta^{\mathbf{r}}\left(\tau_{1}\right)=\Delta^{\mathbf{r}}\left(\tau_{2}\right), \quad \Delta^{\prime}=\Delta^{\mathbf{r}}\left(\tau_{3}\right)=\Delta^{\mathbf{r}}\left(\tau_{4}\right)$. Since $\Delta$ and $\Delta^{\prime}$ have no common factor, the kernel of the matrix $\left[\varphi_{\gamma}\right]$ is generated by the elements $\left[\gamma \mid \tau_{1}\right]-\left[\gamma \mid \tau_{2}\right], \quad\left[\gamma \mid \tau_{2}\right] \Delta^{\prime}-\left[\gamma \mid \tau_{3}\right] \Delta,\left[\gamma \mid \tau_{3}\right]-\left[\gamma \mid \tau_{4}\right]$, which give the description of $\mathfrak{K}_{m, m^{\prime}}^{\mathbf{r}}(\gamma)$ in bi-degree $\leq\left(m, m^{\prime}\right)$. A similar proof applies when there is no horizontal or vertical pair of distinct edges at $\gamma$. This proves the result. 
We use the maps $\left(\varphi_{\gamma}\right)_{\gamma \in \mathcal{T}_{0}^{o}}$ to define

$$
\varphi: \bigoplus_{\gamma \in \mathcal{T}_{0}^{o}} \bigoplus_{\tau \in E_{\gamma}}[\gamma \mid \tau] R_{\left(m, m^{\prime}\right)-\delta(\tau)} \rightarrow \bigoplus_{\gamma \in \mathcal{T}_{0}^{o}}[\gamma] \mathfrak{I}_{m, m^{\prime}}^{\mathbf{r}}(\gamma),
$$

so that we have the following exact sequence:

$$
0 \rightarrow \bigoplus_{\gamma \in \mathcal{T}_{0}^{o}} \mathfrak{K}_{m, m^{\prime}}^{\mathbf{r}}(\gamma) \rightarrow \bigoplus_{\gamma \in \mathcal{T}_{0}^{o}} \bigoplus_{\tau \in E(\gamma)}[\gamma \mid \tau] R_{\left(m, m^{\prime}\right)-\delta(\tau)} \rightarrow \bigoplus_{\gamma \in \mathcal{T}_{0}^{o}}[\gamma] \mathfrak{I}_{m, m^{\prime}}^{\mathbf{r}}(\gamma) \rightarrow 0
$$

Using this exact sequence, we can now identify $\bigoplus_{\gamma \in \mathcal{T}_{1}^{o}}[\gamma] \mathfrak{I}_{m, m^{\prime}}^{\mathrm{r}}(\gamma)$ with the quotient

$$
\bigoplus_{\gamma \in \mathcal{T}_{1}^{o}} \bigoplus_{\tau \in E_{\gamma}}[\gamma \mid \tau] R_{\left(m, m^{\prime}\right)-\delta(\tau)} / \sum_{\gamma \in \mathcal{T}_{1}^{o}} \mathfrak{K}_{m, m^{\prime}}^{\mathrm{r}}(\gamma)
$$

The next proposition uses this identification and Proposition 2.3 to describe more explicitly $H_{0}\left(\mathfrak{I}_{m, m^{\prime}}^{\mathbf{r}}\left(\mathcal{T}^{o}\right)\right)$ :

Proposition 2.4. We have

$$
\begin{aligned}
H_{0}\left(\mathfrak{I}_{m, m^{\prime}}^{\mathbf{r}}\left(\mathcal{T}^{o}\right)\right)= & \bigoplus_{\gamma \in \mathcal{T}_{0}^{o}} \bigoplus_{\tau \in E(\gamma)}[\gamma \mid \tau] R_{\left(m, m^{\prime}\right)-\delta(\tau)} \\
/ & \left(\sum_{\left(\tau, \tau^{\prime}\right) \in P(\gamma)}\left([\gamma \mid \tau]-\left[\gamma \mid \tau^{\prime}\right]\right) R_{\left(m, m^{\prime}\right)-\delta(\tau)}\right. \\
& +\sum_{\tau=\left(\gamma, \gamma^{\prime}\right) \in \mathcal{T}_{1}^{o}}\left([\gamma \mid \tau]-\left[\gamma^{\prime} \mid \tau\right]\right) R_{\left(m, m^{\prime}\right)-\delta(\tau)} \\
& \left.+\sum_{\tau \in E_{h}(\gamma), \tau^{\prime} \in E_{v}(\gamma)}\left([\gamma \mid \tau] \Delta^{\mathbf{r}}\left(\tau^{\prime}\right)-\left[\gamma \mid \tau^{\prime}\right] \Delta^{\mathbf{r}}(\tau)\right) R_{\left(m, m^{\prime}\right)-\delta(\gamma)}\right)
\end{aligned}
$$

Proof. The application

$$
\partial_{1}: \bigoplus_{\tau \in \mathcal{T}_{1}^{o}}[\tau] \mathfrak{I}_{m, m^{\prime}}^{\mathbf{r}}(\tau) \rightarrow \bigoplus_{\gamma \in \mathcal{T}_{0}^{o}}[\gamma] \mathfrak{I}_{m, m^{\prime}}^{\mathbf{r}}(\gamma)
$$

lifts to the application

$$
\begin{aligned}
\tilde{\partial}_{1}: \bigoplus_{\tau \in \mathcal{T}_{1}^{o}}[\tau] R_{\left(m, m^{\prime}\right)-\delta(\tau)} & \rightarrow \bigoplus_{\gamma \in \mathcal{T}_{0}^{o}} \bigoplus_{\tau \in E(\gamma)}[\gamma \mid \tau] R_{\left(m, m^{\prime}\right)-\delta(\tau)} \\
\tau & \mapsto[\gamma \mid \tau]-\left[\gamma^{\prime} \mid \tau\right]
\end{aligned}
$$

so that the image of $\partial_{1}$ lifts in $\bigoplus_{\gamma \in \mathcal{T}_{0}^{o}} \bigoplus_{\tau \in E(\gamma)}[\gamma \mid \tau] R_{\left(m, m^{\prime}\right)-\delta(\tau)}$ to

$$
\operatorname{im} \tilde{\partial}_{1}=\sum_{\tau \in \mathcal{T}_{1}^{o}}\left([\gamma \mid \tau]-\left[\gamma^{\prime} \mid \tau\right]\right) R_{\left(m, m^{\prime}\right)-\delta(\tau)} .
$$

Consequently,

$$
H_{0}\left(\mathfrak{S}_{m, m^{\prime}}^{\mathbf{r}}\left(\mathcal{T}^{o}\right)\right)=\bigoplus_{\gamma \in \mathcal{T}_{0}^{o}} \bigoplus_{\tau \in E(\gamma)}[\gamma \mid \tau] R_{\left(m, m^{\prime}\right)-\delta(\tau)} /\left(\operatorname{im} \tilde{\partial}_{1}+\sum_{\gamma \in \mathcal{T}_{0}^{o}} \mathfrak{K}_{m, m^{\prime}}^{\mathbf{r}}(\gamma)\right),
$$

which yields the desired description of $H_{0}\left(\mathfrak{S}_{m, m^{\prime}}^{\mathbf{r}}(\mathcal{T})\right)$.

In the next proposition, we simplify further the description of $H_{0}\left(\mathfrak{I}_{m, m^{\prime}}^{\mathbf{r}}\left(\mathcal{T}^{o}\right)\right)$ : 


\section{Proposition 2.5.}

$$
\begin{aligned}
H_{0}\left(\mathfrak{I}_{m, m^{\prime}}^{\mathbf{r}}\left(\mathcal{T}^{o}\right)\right)= & \bigoplus_{\rho \in \operatorname{MIS}(\mathcal{T})}[\rho] R_{\left(m, m^{\prime}\right)-\delta(\rho)} \\
/ & \left(\sum_{\gamma \in \mathcal{T}_{0}^{o}}\left(\left[\rho_{v}(\gamma)\right] \Delta_{h}^{\mathbf{r}}(\gamma)-\left[\rho_{h}(\gamma)\right] \Delta_{v}^{\mathbf{r}}(\gamma)\right) R_{\left(m, m^{\prime}\right)-\delta(\gamma)}\right)
\end{aligned}
$$

Proof. Let $B=\bigoplus_{\gamma \in \mathcal{T}_{0}^{o}} \bigoplus_{\tau \in E(\gamma)}[\gamma \mid \tau] R_{\left(m, m^{\prime}\right)-\delta(\tau)}, K=\operatorname{im} \tilde{\partial}_{1}+\sum_{\gamma \in \mathcal{T}_{0}^{o}} \mathfrak{K}_{m, m^{\prime}}^{r, r^{\prime}}(\gamma)$ and

$$
\begin{aligned}
K^{\prime}= & \left(\sum_{\left(\tau, \tau^{\prime}\right) \in P(\gamma)}\left([\gamma \mid \tau]-\left[\gamma \mid \tau^{\prime}\right]\right) R_{\left(m, m^{\prime}\right)-\delta(\tau)}\right. \\
& \left.+\sum_{\tau=\left(\gamma, \gamma^{\prime}\right) \in \mathcal{T}_{1}^{o}}\left([\gamma \mid \tau]-\left[\gamma^{\prime} \mid \tau\right]\right) R_{\left(m, m^{\prime}\right)-\delta(\tau)}\right) .
\end{aligned}
$$

As $K^{\prime} \subset K \subset B$, we have $B / K \equiv\left(B / K^{\prime}\right) /\left(K / K^{\prime}\right)$. Taking the quotient by $K^{\prime}$ means, that we identify the horizontal (resp. vertical) edges which share a vertex. Thus all horizontal (resp. vertical) edges which are contained in a maximal segment $\rho$ of $\mathcal{T}$ are identify to a single element, that we denote $[\rho]$. As $[\gamma \mid \tau]=0$ if $\gamma \in \partial \Omega$, we also have $[\rho]=0$ if the maximal segment $\rho$ intersects the boundary $\partial \Omega$. This yields the desired description of $H_{0}\left(\mathfrak{I}_{m, m^{\prime}}^{\mathbf{r}}(\mathcal{T})\right)$.

Definition 2.6. Let $h_{m, m^{\prime}}^{\mathbf{r}}(\mathcal{T})=\operatorname{dim} H_{0}\left(\mathfrak{I}_{m, m^{\prime}}^{r, r^{\prime}}\left(\mathcal{T}^{o}\right)\right)$.

2.2.2. The 1-homology. We consider now the homology on the edges. We use the property that $H_{1}\left(\mathfrak{R}_{m, m^{\prime}}\left(\mathcal{T}^{o}\right)\right)=0$ (see Proposition D.2 in the appendix).

Proposition 2.7. $H_{1}\left(\mathfrak{S}_{m, m^{\prime}}^{\mathbf{r}}\left(\mathcal{T}^{o}\right)\right)=H_{0}\left(\mathfrak{I}_{m, m^{\prime}}^{\mathbf{r}}\left(\mathcal{T}^{o}\right)\right)$.

Proof. As $H_{0}\left(\Re_{m, m^{\prime}}\left(\mathcal{T}^{o}\right)\right)=0$ and $H_{1}\left(\Re_{m, m^{\prime}}\left(\mathcal{T}^{o}\right)\right)=0$, we deduce from the long exact sequence (see Appendix B)

$\cdots \rightarrow H_{1}\left(\Re_{m, m^{\prime}}\left(\mathcal{T}^{o}\right)\right) \rightarrow H_{1}\left(\mathfrak{S}_{m, m^{\prime}}^{\mathbf{r}}\left(\mathcal{T}^{o}\right)\right) \rightarrow H_{0}\left(\mathfrak{I}_{m, m^{\prime}}^{\mathbf{r}}\left(\mathcal{T}^{o}\right)\right) \rightarrow H_{0}\left(\Re_{m, m^{\prime}}\left(\mathcal{T}^{o}\right)\right) \rightarrow \cdots$

that $H_{1}\left(\mathfrak{S}_{m, m^{\prime}}^{\mathbf{r}}\left(\mathcal{T}^{o}\right)\right) \sim H_{0}\left(\mathfrak{I}_{m, m^{\prime}}^{\mathbf{r}}\left(\mathcal{T}^{o}\right)\right)$.

2.2.3. The 2-homology. Finally, the homology on the 2 -faces will give us information on the spline space $\mathcal{S}_{m, m^{\prime}}^{\mathbf{r}}(\mathcal{T})$.

We have the following result (proved in Proposition D.3 in the appendix):

Proposition 2.8. $H_{2}\left(\Re_{m, m^{\prime}}\left(\mathcal{T}^{o}\right)\right)=R_{m, m^{\prime}}$.

The following proposition relates the spline space $\mathcal{S}_{m, m^{\prime}}^{\mathbf{r}}(\mathcal{T})$ to an homology module.

Proposition 2.9. $H_{2}\left(\mathfrak{S}_{m, m^{\prime}}^{\mathbf{r}}\left(\mathcal{T}^{o}\right)\right)=\operatorname{ker} \partial_{2}=\mathcal{S}_{m, m^{\prime}}^{\mathbf{r}}(\mathcal{T})$

Proof. An element of $H_{2}\left(\mathfrak{S}_{m, m^{\prime}}^{\mathbf{r}}\left(\mathcal{T}^{o}\right)\right)=\operatorname{ker} \bar{\partial}_{2}$ is a collection of polynomials $\left(p_{\sigma}\right)_{\sigma \in \mathcal{T}_{2}}$ with $p_{\sigma} \in R_{m, m^{\prime}}$ and $p_{\sigma} \equiv p_{\sigma^{\prime}} \bmod \mathfrak{I}_{\tau}^{\mathbf{r}}(\tau)$ if $\sigma$ and $\sigma^{\prime}$ share the (internal) edge $\tau$. By Lemma 1.6, this implies that the piecewise polynomial function which is $p_{\sigma}$ on $\sigma$ and $p_{\sigma^{\prime}}$ on $\sigma^{\prime}$ is of class $C^{\mathbf{r}}$ across the edge $\tau$. As this is true for all interior edges, $\left(p_{\sigma}\right)_{\sigma \in \mathcal{T}_{2}} \in \operatorname{ker} \underline{\partial}_{2}$ is a piecewise polynomial function of $R_{m, m^{\prime}}$ which is of class $C^{\mathbf{r}}$, that is an element of $\mathcal{S}_{m, m^{\prime}}^{\mathbf{r}}(\mathcal{T})$. 
3. LOWER AND UPPER BOUNDS ON THE DIMENSION

In this section, are the main results on the dimension of the spline space $\mathcal{S}_{m, m^{\prime}}^{\mathbf{r}}(\mathcal{T})$.

Theorem 3.1. Let $\mathcal{T}$ be a T-mesh and let $\mathbf{r}$ be a smoothness distribution on $\mathcal{T}$. Then

$$
\begin{aligned}
\operatorname{dim} \mathcal{S}_{m, m^{\prime}}^{\mathbf{r}}(\mathcal{T}) & =\sum_{\sigma \in \mathcal{T}_{2}}(m+1)\left(m^{\prime}+1\right) \\
& -\sum_{\tau \in \mathcal{T}_{1}^{o, h}}(m+1)(\mathbf{r}(\tau)+1)-\sum_{\tau \in \mathcal{T}_{1}^{o, v}}\left(m^{\prime}+1\right)(\mathbf{r}(\tau)+1) \\
& +\sum_{\gamma \in \mathcal{T}_{0}^{o}}\left(\mathbf{r}_{h}(\gamma)+1\right)\left(\mathbf{r}_{v}(\gamma)+1\right) \\
& +h_{m, m^{\prime}}^{\mathbf{r}}(\mathcal{T})
\end{aligned}
$$

where $h_{m, m^{\prime}}^{\mathbf{r}}(\mathcal{T})=\operatorname{dim} H_{0}\left(\mathfrak{I}_{m, m^{\prime}}^{\mathbf{r}}\left(\mathcal{T}^{o}\right)\right)$.

Proof. The complex

$$
\begin{aligned}
\mathfrak{S}_{m, m^{\prime}}^{\mathbf{r}}\left(\mathcal{T}^{o}\right): \bigoplus_{\sigma \in \mathcal{T}_{2}}[\sigma] R_{m, m^{\prime}} & \longrightarrow \bigoplus_{\tau \in \mathcal{T}_{1}^{o}}[\tau] R_{m, m^{\prime}} / \mathfrak{I}_{m, m^{\prime}}^{\mathbf{r}}(\tau) \\
& \longrightarrow \bigoplus_{\gamma \in \mathcal{T}_{0}^{o}}[\gamma] R_{m, m^{\prime}} / \mathfrak{I}_{m, m^{\prime}}^{\mathbf{r}}(\gamma) \longrightarrow 0
\end{aligned}
$$

induces the following relations:

$$
\begin{aligned}
\operatorname{dim} & \left(\bigoplus_{\sigma \in \mathcal{T}_{2}}[\sigma] R_{m, m^{\prime}}\right)-\operatorname{dim}\left(\bigoplus_{\tau \in \mathcal{T}_{1}^{o}}[\tau] R_{m, m^{\prime}} / \mathfrak{I}_{m, m^{\prime}}^{r, r^{\prime}}(\tau)\right)+\operatorname{dim}\left(\bigoplus_{\gamma \in \mathcal{T}_{0}^{o}}[\gamma] R_{m, m^{\prime}} / \mathfrak{I}_{m, m^{\prime}}^{\mathrm{r}}(\gamma)\right) \\
& =\operatorname{dim}\left(H_{2}\left(\mathfrak{S}_{m, m^{\prime}}^{\mathrm{r}}\left(\mathcal{T}^{o}\right)\right)\right)-\operatorname{dim}\left(H_{1}\left(\mathfrak{S}_{m, m^{\prime}}^{r, r}\left(\mathcal{T}^{o}\right)\right)\right)+\operatorname{dim}\left(H_{0}\left(\mathfrak{S}_{m, m^{\prime}}^{\mathrm{r}}\left(\mathcal{T}^{o}\right)\right)\right) .
\end{aligned}
$$

As $H_{2}\left(\mathfrak{S}_{m, m^{\prime}}^{\mathbf{r}}\left(\mathcal{T}^{o}\right)\right)=\mathcal{S}_{m, m^{\prime}}^{\mathbf{r}}(\mathcal{T}), H_{0}\left(\mathfrak{S}_{m, m^{\prime}}^{\mathbf{r}}\left(\mathcal{T}^{o}\right)\right)=0$ and $H_{1}\left(\mathfrak{S}_{m, m^{\prime}}^{\mathbf{r}}\left(\mathcal{T}^{o}\right)\right)=$ $H_{0}\left(\mathfrak{I}_{m, m^{\prime}}^{\mathbf{r}}\left(\mathcal{T}^{o}\right)\right)$, we deduce that

$$
\begin{aligned}
\operatorname{dim} \mathcal{S}_{m, m^{\prime}}^{\mathbf{r}}(\mathcal{T}) & =\operatorname{dim}\left(\bigoplus_{\sigma \in \mathcal{T}_{2}}[\sigma] R_{m, m^{\prime}}\right)-\operatorname{dim}\left(\bigoplus_{\tau \in \mathcal{T}_{1}^{o}}[\tau] R_{m, m^{\prime}} / \mathfrak{I}_{m, m^{\prime}}^{\mathbf{r}}(\tau)\right) \\
& +\operatorname{dim}\left(\bigoplus_{\gamma \in \mathcal{T}_{0}^{o}}[\gamma] R_{m, m^{\prime}} / \mathfrak{I}_{m, m^{\prime}}^{\mathbf{r}}(\gamma)\right)+\operatorname{dim}\left(H_{0}\left(\mathfrak{I}_{m, m^{\prime}}^{r, r^{\prime}}(\mathcal{T})\right)\right),
\end{aligned}
$$

which yields the dimension formula (3.1) using Lemma 1.5.

As an immediate corollary of this theorem and of Proposition 2.5], we deduce the following result:

Corollary 3.2. If the T-mesh $\mathcal{T}$ has no maximal interior segments, then $h_{m, m^{\prime}}^{\mathbf{r}}(\mathcal{T})$ $=0$.

In the case of a constant smoothness distribution, Theorem 3.1 is written as follows:

Theorem 3.3. Let $\mathcal{T}$ be a T-mesh and let $\mathbf{r}=\left(r, r^{\prime}\right)$ be a constant smoothness distribution on $\mathcal{T}$. Then

$$
\begin{aligned}
\operatorname{dim} \mathcal{S}_{m, m^{\prime}}^{\mathbf{r}}(\mathcal{T}) & =(m+1)\left(m^{\prime}+1\right) f_{2} \\
& -\left((m+1)\left(r^{\prime}+1\right) f_{1}^{h}+\left(m^{\prime}+1\right)(r+1) f_{1}^{v}\right) \\
& +(r+1)\left(r^{\prime}+1\right) f_{0} \\
& +h_{m, m^{\prime}}^{\mathbf{r}}(\mathcal{T}),
\end{aligned}
$$


where

- $f_{2}$ is the number of 2-faces $\in \mathcal{T}_{2}$,

- $f_{1}^{h}\left(\right.$ resp. $\left.f_{1}^{v}\right)$ is the number of horizontal (resp. vertical) interior edges $\in \mathcal{T}_{1}^{o}$,

- $f_{0}$ is the number of interior vertices $\in \mathcal{T}_{0}^{o}$,

- $h_{m, m^{\prime}}^{\mathbf{r}}(\mathcal{T})=\operatorname{dim} H_{0}\left(\mathfrak{I}_{m, m^{\prime}}^{\mathbf{r}}\left(\mathcal{T}^{o}\right)\right)$.

We are now going to bound $h_{m, m^{\prime}}^{\mathbf{r}}(\mathcal{T})$ for general T-meshes.

Definition 3.4. Let $\iota$ be an ordering of $\operatorname{MIS}(\mathcal{T})$ that is a map from $\operatorname{MIS}(\mathcal{T})$ to $\mathbb{N}$. For $\rho \in \operatorname{MiS}(\mathcal{T})$, let $\Gamma_{\iota}(\rho)$ be the set of vertices $\gamma$ of $\rho$ which are not on a maximal interior segment $\rho^{\prime} \in \operatorname{MIS}(\mathcal{T})$ with $\iota\left(\rho^{\prime}\right)>\iota(\rho)$. The number of elements of $\Gamma_{\iota}(\rho)$ is denoted $\lambda_{\iota}(\rho)$.

We define now the weight of a maximal interior segment.

Definition 3.5. For $\rho \in \operatorname{MIS}(\mathcal{T})$, let

- $\omega_{\iota}(\rho)=\sum_{\gamma \in \Gamma_{\iota}}\left(m-\mathbf{r}_{v}(\gamma)\right)$ if $\rho \in \operatorname{MIS}_{h}(\mathcal{T})$.

- $\omega_{\iota}(\rho)=\sum_{\gamma \in \Gamma_{\iota}}\left(m^{\prime}-\mathbf{r}_{h}(\gamma)\right)$ if $\rho \in \operatorname{MIS}_{v}(\mathcal{T})$.

We called it the weight of $\rho$.

As in the usual spline terminology, for an interior point $\gamma \in \mathcal{T}_{0}^{o}$, we call $\gamma$ $m-\mathbf{r}_{h}(\gamma)$ (resp. $m^{\prime}-\mathbf{r}_{h}(\gamma)$ ) the horizontal (resp. vertical) multiplicity of $\gamma$.

If $\rho$ is horizontal (resp. vertical), the weight of $\rho$ is the sum of the vertical (resp. horizontal) multiplicities of the vertices $\gamma \in \Gamma_{\iota}(\rho)$.

Notice that if $\mathbf{r}=\left(r, r^{\prime}\right)$ is a constant smoothness distribution on $\mathcal{T}$, then $\omega_{\iota}(\rho)=$ $(m-r) \lambda(\rho)$ for $\rho \in \operatorname{MIS}_{h}(\mathcal{T})\left(\operatorname{resp} . \omega_{\iota}(\rho)=\left(m^{\prime}-r^{\prime}\right) \lambda(\rho)\right.$ for $\left.\rho \in \operatorname{MIS}_{v}(\mathcal{T})\right)$.

Example 3.6. We consider the T-mesh of Example 1.9 with $\left(m, m^{\prime}\right)=(2,2)$, the constant smoothness distribution $\mathbf{r}=(1,1)$ and the ordering of the maximal interior segments $\iota\left(\rho_{i}\right)=i$ for $i=1, \ldots, 4$. Then we have

- $\omega_{\iota}\left(\rho_{1}\right)=(2-1) \times 2=2$,

- $\omega_{\iota}\left(\rho_{2}\right)=(2-1) \times 2=2$,

- $\omega_{\iota}\left(\rho_{3}\right)=(2-1) \times 3=3$,

- $\omega_{\iota}\left(\rho_{4}\right)=(2-1) \times 3=3$,

since the multiplicity of a vertex is $2-1=1$ and the interior points of $\rho_{1}, \rho_{2}$ are not in $\Gamma_{\iota}\left(\rho_{1}\right)$ or $\Gamma_{\iota}\left(\rho_{2}\right)$.

In the following, we will drop the index $\iota$ to simplify the notation, assuming that the ordering $\iota$ is fixed. Then we have the following theorem:

Theorem 3.7. Let $\mathcal{T}$ be a T-mesh and let $\mathbf{r}$ be a smoothness distribution on $\mathcal{T}$. Then

$$
\begin{aligned}
0 \leq h_{m, m^{\prime}}^{\mathbf{r}}(\mathcal{T}) & \leq \sum_{\rho \in \operatorname{MIS}_{h}(\mathcal{T})}(m+1-\omega(\rho))_{+} \times\left(m^{\prime}-\mathbf{r}(\rho)\right) \\
& +\sum_{\rho \in \operatorname{MIS}_{v}(\mathcal{T})}(m-\mathbf{r}(\rho)) \times\left(m^{\prime}+1-\omega(\rho)\right)_{+} .
\end{aligned}
$$

Proof. Let $\rho_{1}, \ldots, \rho_{l}$ be the maximal interior segments of $\mathcal{T}$. By Proposition 2.5. $h_{m, m^{\prime}}^{\mathbf{r}}(\mathcal{T})$ is the dimension of the quotient in bi-degree $\leq\left(m, m^{\prime}\right)$ of the module $M:=\bigoplus_{i=1}^{l}\left[\rho_{i}\right] R$ by the module $K$ generated by the following relations: for each vertex $\gamma \in \mathcal{T}_{0}^{o}$ which is on a maximal interior segment, 
- $\Delta^{\mathbf{r}}\left(\rho_{j}\right)\left[\rho_{i}\right]-\Delta^{\mathbf{r}}\left(\rho_{i}\right)\left[\rho_{j}\right]$ if $\gamma$ is the intersection of the maximal interior segments $\rho_{i}$ and $\rho_{j}$,

- $\Delta^{\mathbf{r}}(\rho)\left[\rho_{i}\right]$ if $\gamma$ is the intersection of the maximal interior segment $\rho_{i}$ with another maximal segment $\rho$ intersecting $\partial \Omega$.

To compute the dimension of $M / K$ in bi-degree $\leqslant\left(m, m^{\prime}\right)$, we use a graduation on $M$ given by the indices of the segments. For $r:=\sum_{i} p_{i}\left[\rho_{i}\right] \in M$ (with $p_{i} \in$ $\left.R_{\left(m, m^{\prime}\right)-\delta\left(\rho_{i}\right)}\right)$, let $\operatorname{In}(m)$ be the element $p_{i_{0}}\left[\rho_{i_{0}}\right]$ where $i_{0}$ is the minimal index such that $p_{i} \neq 0$. We denote it by $\operatorname{In}(r)$ and called it the initial of $r$. Let $\operatorname{In}(K)=$ $\{\operatorname{In}(k) \mid k \in K\}$. The dimension $h_{m, m^{\prime}}^{\mathbf{r}}(\mathcal{T})$ is then

$$
h_{m, m^{\prime}}^{\mathbf{r}}(\mathcal{T})=\operatorname{dim}(M / K)=\operatorname{dim}(M / \operatorname{In}(K)) .
$$

Notice that $\operatorname{In}(K)$ contains the multiples in bi-degree $\leqslant\left(m, m^{\prime}\right)$ of

- $\Delta^{\mathbf{r}}\left(\rho_{j}\right)\left[\rho_{i}\right]$ if $\gamma$ is the intersection of two maximal interior segments $\rho_{i}$ and $\rho_{j}$ with $i>j$,

- $\Delta^{\mathbf{r}}(\rho)\left[\rho_{i}\right]$ if $\gamma$ is the intersection of the maximal interior segment $\rho_{i}$ with a maximal segment $\rho$ intersecting $\Omega$.

Let $L_{i}$ be the vector space spanned by these initials in bi-degree $\leqslant\left(m, m^{\prime}\right)$, which are multiples of $\left[\rho_{i}\right]$. By definition, for each $\gamma \in \Gamma\left(\rho_{i}\right)$, we have a generator $\Delta^{\mathbf{r}}(\rho)\left[\rho_{i}\right]$ in $L_{i}$ for $\{\gamma\}=\rho_{i} \cap \rho$. By Proposition 1.8, $L_{i}$ is of dimension

- $\min \left(m+1, \omega\left(\rho_{i}\right)\right) \times\left(m^{\prime}-\mathbf{r}\left(\rho_{i}\right)\right)$ if $\rho_{i} \in \operatorname{MIS}_{h}(\mathcal{T})$,

- $\min \left(m^{\prime}+1, \omega\left(\rho_{i}\right)\right) \times\left(m-\mathbf{r}\left(\rho_{i}\right)\right)$ if $\rho_{i} \in \operatorname{MIS}_{v}(\mathcal{T})$.

Thus the dimension of $\left[\rho_{i}\right] R_{\left(m, m^{\prime}\right)-\delta\left(\rho_{i}\right)} / L_{i}$ is

- $\left(m+1-\omega\left(\rho_{i}\right)\right)_{+} \times\left(m^{\prime}-\mathbf{r}\left(\rho_{i}\right)\right)$ if $\rho_{i} \in \operatorname{MIS}_{h}(\mathcal{T})$,

- $\left(m-\mathbf{r}\left(\rho_{i}\right)\right) \times\left(m^{\prime}+1-\omega\left(\rho_{i}\right)\right)_{+}$if $\rho_{i} \in \operatorname{MIS}_{v}(\mathcal{T})$.

As $\operatorname{In}(K) \supset \sum_{i} L_{i}$, we have

$$
\begin{aligned}
h_{m, m^{\prime}}^{\mathbf{r}}(\mathcal{T}) & =\operatorname{dim}(M / \operatorname{In}(K)) \\
& \leq \operatorname{dim}\left(\left[\rho_{i}\right] R_{\left(m, m^{\prime}\right)-\delta\left(\rho_{i}\right)} /\left(\sum_{i} L_{i}\right)\right)=\sum_{i} \operatorname{dim}\left(\left[\rho_{i}\right] R_{\left(m, m^{\prime}\right)-\delta\left(\rho_{i}\right)} / L_{i}\right) .
\end{aligned}
$$

This gives the announced bound on $h_{m, m^{\prime}}^{\mathbf{r}}(\mathcal{T})$, using the previous computation of $\operatorname{dim}\left(\left[\rho_{i}\right] R_{\left(m, m^{\prime}\right)-\delta\left(\rho_{i}\right)} / L_{i}\right)$.

Definition 3.8. The T-mesh $\mathcal{T}$ with a smoothness distribution $\mathbf{r}$ is $\left(k, k^{\prime}\right)$-weighted if

- $\forall \rho \in \operatorname{MIS}_{h}(\mathcal{T}), \omega(\rho) \geq k$,

- $\forall \rho \in \operatorname{MIS}_{v}(\mathcal{T}), \omega(\rho) \geq k^{\prime}$;

Theorem 3.9. Let $\mathcal{T}$ be a $T$-mesh and let $\mathbf{r}$ be a smoothness distribution on $\mathcal{T}$. If $\mathcal{T}$ is $\left(m+1, m^{\prime}+1\right)$-weighted, then $h_{m, m^{\prime}}^{\mathbf{r}}(\mathcal{T})=0$.

Proof. By definition, $\forall \rho \in \operatorname{MIS}_{h}(\mathcal{T}), \omega(\rho) \geq m+1$ (i.e. $\left.(m+1-\omega(\rho))_{+}=0\right)$ and $\forall \rho \in \operatorname{MIS}_{v}(\mathcal{T}), \omega(\rho) \geq m^{\prime}+1$ (i.e. $\left.\left(m^{\prime}+1-\omega(\rho)\right)_{+}=0\right)$. By Theorem 3.7, we directly deduce that $h_{m, m^{\prime}}^{\mathbf{r}}(\mathcal{T})=0$.

Here is a direct corollary which generalizes a result in [16]:

Corollary 3.10. Suppose that the end points of a maximal interior segment $\rho \in$ $\operatorname{MIS}(\mathcal{T})$ are in $\Gamma(\rho)$. If for each horizontal (resp. vertical) maximal interior segment $\rho \in \operatorname{MIS}(\mathcal{T})$, the sum of the vertical (resp. horizontal) multiplicities of the end points 
and of the vertices of $\rho$ on a maximal segment connected to the boundary is greater than or equal to $m+1$ (resp. $\left.m^{\prime}+1\right)$, then $h_{m, m^{\prime}}^{\mathbf{r}}(\mathcal{T})=0$.

Proof. Let $\rho$ be a maximal interior segment of $\mathcal{T}$. By hypothesis, the end points of $\rho$ are in $\Gamma(\rho)$. As any point $\gamma \in \rho$ which is also on a maximal segment connected to the boundary is in $\Gamma(\rho)$, the hypothesis implies that $\omega(\rho) \geq m+1$ if $\rho$ is horizontal and $\omega(\rho) \geq m^{\prime}+1$ if $\rho$ is vertical. We deduce by Theorem 3.7 that $h_{m, m^{\prime}}^{\mathbf{r}}(\mathcal{T})=0$.

Another case where $h_{m, m^{\prime}}^{\mathbf{r}}(\mathcal{T})$ is known is described in the next proposition.

Proposition 3.11. If $\forall \rho \in \operatorname{MIS}_{h}(\mathcal{T}), \omega(\rho) \leq m+1$ and $\forall \rho \in \operatorname{MIS}_{v}(\mathcal{T}), \omega(\rho) \leq$ $m^{\prime}+1$, then

$$
\begin{aligned}
h_{m, m^{\prime}}^{\mathbf{r}}(\mathcal{T}) & =\sum_{\rho \in \operatorname{MIS}_{h}(\mathcal{T})}(m+1-\omega(\rho))_{+} \times\left(m^{\prime}-\mathbf{r}(\rho)\right) \\
& +\sum_{\rho \in \operatorname{MIS}_{v}(\mathcal{T})}(m-\mathbf{r}(\rho)) \times\left(m^{\prime}+1-\omega(\rho)\right)_{+} .
\end{aligned}
$$

Proof. In the case where $\forall \rho \in \operatorname{MIS}_{h}(\mathcal{T}), \omega(\rho) \leq m+1$ and $\forall \rho \in \operatorname{MIS}_{v}(\mathcal{T}), \omega(\rho) \leq$ $m^{\prime}+1$, Proposition 1.8 implies that there is no relations in bi-degree $\leqslant\left(m, m^{\prime}\right)$ of the monomial multiples of $\Delta^{\mathbf{r}}\left(\rho_{j}\right)\left[\rho_{i}\right], \Delta^{\mathbf{r}}(\rho)\left[\rho_{i}\right]$ for $i=1, \ldots, l, j<i$ using the same notation as in the proof of Theorem 3.7. This implies that $\operatorname{In}(K)=\bigoplus_{i} L_{i}$, which shows that

$$
h_{m, m^{\prime}}^{\mathbf{r}}(\mathcal{T})=\operatorname{dim}(M / \operatorname{In}(K))=\sum_{i} \operatorname{dim}\left(R_{\left(m, m^{\prime}\right)-\delta\left(\rho_{i}\right)}\left[\rho_{i}\right] / L_{i}\right) .
$$

Thus the equality (3.3) holds.

As a corollary, we have the following result for constant smoothness distribution:

Theorem 3.12. Let $\mathcal{T}$ be a T-mesh and let $\mathbf{r}=\left(r, r^{\prime}\right)$ be a constant smoothness distribution on $\mathcal{T}$. Then

$$
\begin{aligned}
0 \leq h_{m, m^{\prime}}^{\mathbf{r}}(\mathcal{T}) & \leq \sum_{\rho \in \operatorname{MIS}_{h}(\mathcal{T})}(m+1-(m-r) \lambda(\rho))_{+} \times\left(m^{\prime}-r^{\prime}\right) \\
& +\sum_{\rho \in \operatorname{MIS}_{v}(\mathcal{T})}(m-r) \times\left(m^{\prime}+1-\left(m^{\prime}-r^{\prime}\right) \lambda(\rho)\right)_{+} .
\end{aligned}
$$

Moreover, equality holds in the following cases:

- $\forall \rho \in \operatorname{MIS}_{h}(\mathcal{T}),(m-r) \lambda(\rho) \geq m+1$ and $\forall \rho \in \operatorname{MIS}_{v}(\mathcal{T}),\left(m^{\prime}-r^{\prime}\right) \lambda(\rho) \geq$ $m^{\prime}+1$;

- $\forall \rho \in \operatorname{MIS}_{h}(\mathcal{T}),(m-r) \lambda(\rho) \leq m+1$ and $\forall \rho \in \operatorname{MIS}_{v}(\mathcal{T}),\left(m^{\prime}-r^{\prime}\right) \lambda(\rho) \leq$ $m^{\prime}+1$.

\section{HierarchicAl T-Meshes}

We consider now a special family of T-meshes, which can be defined by recursive subdivision from an initial rectangular domain $\Omega$. Their study is motivated by practical applications, where local refinement of tensor-product spline spaces are considered e.g. in isogeometric analysis [14].

Definition 4.1. A hierarchical T-mesh is either the initial axis-aligned rectangle $\Omega$ or obtained from a hierarchical T-mesh by splitting a cell along a vertical or horizontal line. 
A hierarchical T-mesh will also be called a T-subdivision. It can be represented by a subdivision tree where the nodes are the cells obtained during the subdivision and the children of a cell $\sigma$ are the cells obtained by subdividing $\sigma$.

In a hierarchical T-mesh $\mathcal{T}$, the maximal interior segments are naturally ordered in the way they appear during the subdivision process. This is the ordering $\iota$ that we will consider hereafter. Notice that a maximal interior segment $\rho$ is transformed by a split either into a maximal segment which intersects $\partial \Omega$ or into a larger maximal interior segment with a larger weight.

We remark that in a hierarchical T-mesh, if $\rho_{i}$ is blocking $\rho_{j}$ then $i<j$.

Example 4.2. Here are an hierarchical T-mesh (case a) and a non-hierarchical T-mesh (case b):

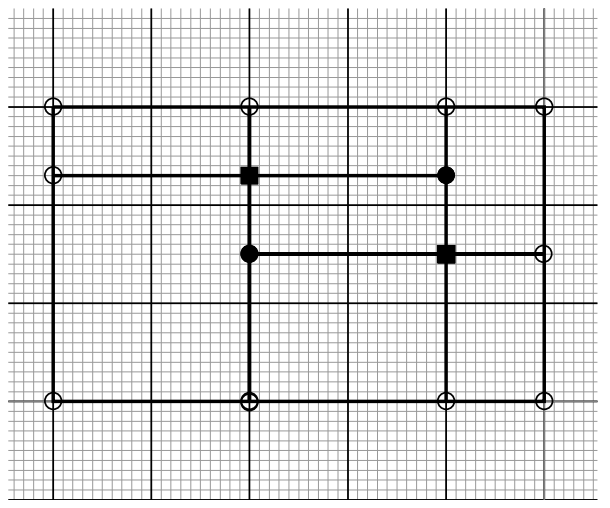

(a)

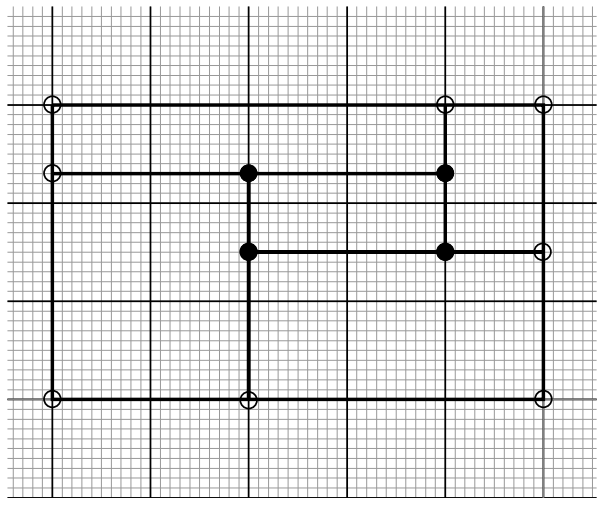

(b)

4.1. Dimension formula for hierarchical T-meshes. As a corollary of Theorem 3.7 we deduce the following result, also proved in [6], [16], [13]:

Proposition 4.3. Let $\mathcal{T}$ be a hierarchical T-mesh and let $\mathbf{r}=\left(r, r^{\prime}\right)$ be a constant smoothness distribution on $\mathcal{T}$. For $m \geq 2 r+1$ and $m^{\prime} \geq 2 r^{\prime}+1$, we have $h_{m, m^{\prime}}^{r, r^{\prime}}(\mathcal{T})=$ 0 .

Proof. We order the maximal interior segments in the way they appear during the subdivision. If a segment $\rho_{i} \in \operatorname{MIS}(\mathcal{T})$ is blocking $\rho_{j} \in \operatorname{MIS}(\mathcal{T})$, we must have $i<j$. This shows that the end points of $\rho_{i}$ are in $\Gamma\left(\rho_{i}\right)$. Thus, $\lambda\left(\rho_{i}\right) \geq 2$.

As $m \geq 2 r+1$, we have

$$
(m-r) \lambda\left(\rho_{i}\right) \geq 2(m-r) \geq m+(m-2 r) \geq m+1 .
$$

Thus, $\left(m+1-(m-r) \lambda\left(\rho_{i}\right)\right)_{+}=0$. Similarly $\left(m^{\prime}+1-\left(m^{\prime}-r^{\prime}\right) \lambda\left(\rho_{i}\right)\right)_{+}=0$ holds since $m^{\prime} \geq 2 r^{\prime}+1$. By Theorem 3.7, we deduce that $h_{m, m^{\prime}}^{\mathbf{r}}(\mathcal{T})=0$.

Theorem 3.9 leads us to the following construction rule of a T-subdivision $\mathcal{T}$ for which $h_{m, m^{\prime}}^{\mathbf{r}}(\mathcal{T})=0$.

Algorithm $4.4\left(\left(k, k^{\prime}\right)\right.$-weighted subdivision rule).

For each 2-face $\sigma$ of a T-mesh to be subdivided:

(1) Split $\sigma$ with the new edge $\tau$;

(2) If the edge $\tau$ does not extend an existing segment, extend $\tau$ (on one side and/or the other) so that the maximal segment containing $\tau$ is either intersecting $\partial \Omega$ or horizontal (resp. vertical) and of weight $\geq k$ (resp. $\left.\geq k^{\prime}\right)$. 
If such a rule is applied in the construction of a T-subdivision,

- either a new maximal interior segment is constructed so that its weight is $\geq k$ if it is a horizontal maximal interior segment (resp. $\geq k^{\prime}$ it is a vertical maximal interior segment),

- or an existing maximal interior segment is extended and its weight is also increased,

- or a maximal segment intersecting $\partial \Omega$ is constructed.

In all cases, if we start with a $\left(k, k^{\prime}\right)$-weighted T-mesh, we obtain a new T-mesh, which is also $\left(k, k^{\prime}\right)$-weighted.

By Theorem 3.9, if $k \geq m+1$ and $k^{\prime} \geq m^{\prime}+1$ then $h_{m, m^{\prime}}^{\mathbf{r}}(\mathcal{T})=0$ and the dimension of $\operatorname{dim} \mathcal{S}_{m, m^{\prime}}^{\mathbf{r}}(\mathcal{T})$, given by formula (3.1), depends only on the number of cells, interior segments and interior vertices of $\mathcal{T}$. From this analysis, we deduce the dimension formula of the space of Locally Refined splines described in [9].

\section{EXAMPLES}

In this section, we analyse the dimension formula of spline spaces of small bidegree and small constant smoothness distribution $\mathbf{r}=\left(r, r^{\prime}\right)$ on a T-mesh $\mathcal{T}$.

5.1. Bilinear $C^{0,0} \mathbf{T}$-splines. We consider first piecewise bilinear polynomials on $\mathcal{T}$ which are continuous, that is, $m=m^{\prime}=1$ and $r=r^{\prime}=0$. By Proposition 4.3, we have $h_{1,1}^{0,0}(\mathcal{T})=0$. Using Theorem 3.1 and Lemma A.1 in the appendix, we obtain:

$$
\operatorname{dim} \mathcal{S}_{1,1}^{0,0}(\mathcal{T})=4 f_{2}-2 f_{1}^{o}+f_{0}^{o}=f_{0}^{+}+f_{0}^{b}
$$

5.2. Biquadratic $C^{1,1}$ T-splines. Let us consider now the set of piecewise biquadratic functions on a T-mesh $\mathcal{T}$, which are $C^{1}$. For $m=m^{\prime}=2$ and $r=r^{\prime}=1$, Theorem 3.1 and Lemma A.1 again yield

$(5.2) \operatorname{dim} \mathcal{S}_{2,2}^{1,1}(\mathcal{T})=9 f_{2}-6 f_{1}^{o}+4 f_{0}^{o}+h_{2,2}^{1,1}(\mathcal{T})=f_{0}^{+}-\frac{1}{2} f_{0}^{T}+\frac{3}{2} f_{0}^{b}+3+h_{2,2}^{1,1}(\mathcal{T})$.

If the T-mesh $\mathcal{T}$ is $(3,3)$-weighted, then by Theorem 3.9 we have a $h_{2,2}^{1,1}(\mathcal{T})=0$, but this is not always the case.

Example 5.1. Here is an example where $h_{2,2}^{1,1}(\mathcal{T})=1$ by Proposition 3.11, since there is one maximal interior segment $\rho$ with $\omega(\rho)=2-1+2-1=2$ :

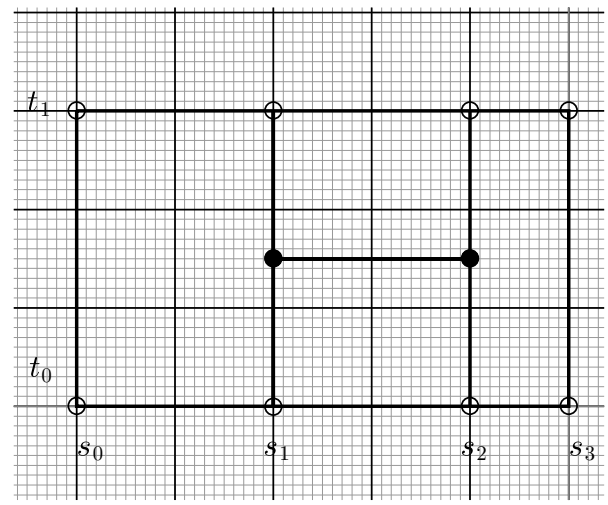


The dimension of $\mathcal{S}_{2,2}^{1,1}(\mathcal{T})$ is $9 \times 4-6 \times 5+4 \times 2+h_{2,2}^{1,1}(\mathcal{T})=14+1=15$. Notice that the dimension is the same without the (horizontal) interior segment. Thus a basis of $\mathcal{S}_{2,2}^{1,1}(\mathcal{T})$ is the tensor product B-spline basis corresponding to the nodes $s_{0}, s_{0}, s_{0}, s_{1}, s_{2}, s_{3}, s_{3}, s_{3}$ in the horizontal direction and the nodes $t_{0}, t_{0}, t_{0}, t_{1}, t_{1}, t_{1}$ in the vertical direction.

Example 5.2. Here is another example. We subdivide the T-mesh $\mathcal{T}_{1}$ to obtain the second T-mesh $\mathcal{T}_{2}$ :
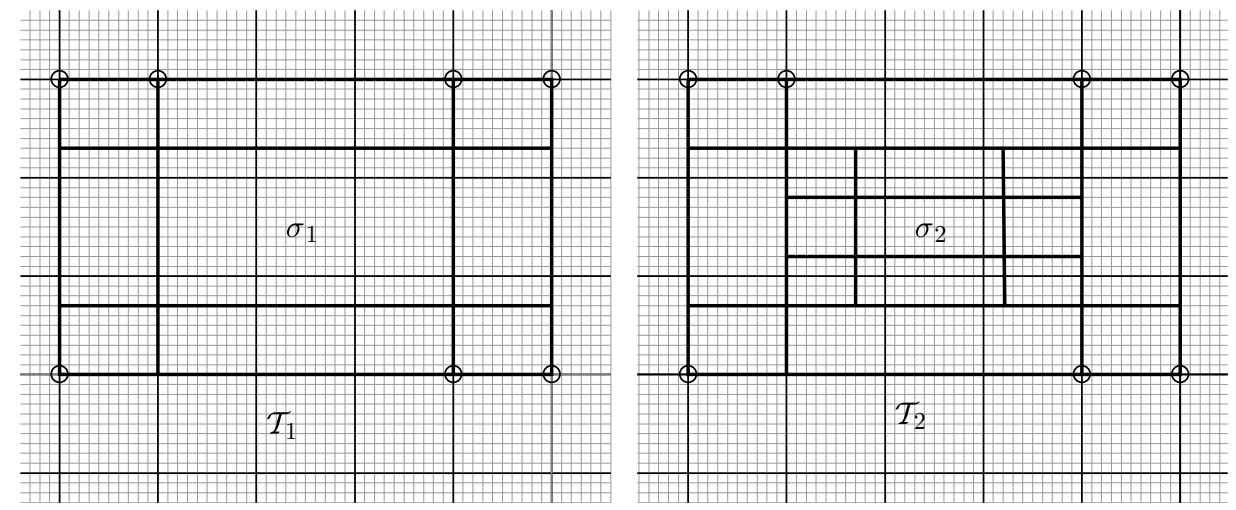

Doing this, we increase the number of cells by $9-1=8$, the number of interior edges by $24-4=20$, the number of interior points by $16-4=12$. The dimension of the spline space increases by $9 \times 8-6 \times 20+4 \times 12+h_{2,2}^{1,1}\left(\mathcal{T}_{2}\right)-h_{2,2}^{1,1}\left(\mathcal{T}_{1}\right)=$ $h_{2,2}^{1,1}\left(\mathcal{T}_{2}\right)-h_{2,2}^{1,1}\left(\mathcal{T}_{1}\right)$. Since there is no maximal interior segment in $\mathcal{T}_{1}$, by Corollary 3.2 we have $h_{2,2}^{1,1}\left(\mathcal{T}_{1}\right)=0$. Choosing a proper ordering of the interior segments, we deduce by Theorem 3.1 that $h_{2,2}^{1,1}\left(\mathcal{T}_{2}\right) \leq 1$. Suppose that $\sigma_{1}=\left[a_{0}, a_{3}\right] \times\left[b_{0}, b_{3}\right]$ and $\sigma_{2}=\left[a_{1}, a_{2}\right] \times\left[b_{1}, b_{2}\right]$. For $u_{0} \leq u_{1} \leq u_{2} \leq u_{3} \in \mathbb{R}$, let $N\left(u ; u_{0}, u_{1}, u_{2}, u_{3}\right)$ be the B-spline basis function in the variable $u$ of degree 2 associated to the nodes $u_{0}, \ldots, u_{3}$ (see [5]). Then the piecewise polynomial function

$$
N\left(s ; a_{0}, a_{1}, a_{2}, a_{3}\right) \times N\left(t ; b_{0}, b_{1}, b_{2}, b_{3}\right)
$$

is an element of $\mathcal{S}_{2,2}^{1,1}\left(\mathcal{T}_{2}\right)$, with support in $\sigma_{1}$. It is not in $\mathcal{S}_{2,2}^{1,1}\left(\mathcal{T}_{1}\right)$, since the function is not polynomial on $\sigma_{1}$. Thus we have $\operatorname{dim} \mathcal{S}_{2,2}^{1,1}\left(\mathcal{T}_{2}\right)=\operatorname{dim} \mathcal{S}_{2,2}^{1,1}\left(\mathcal{T}_{1}\right)+1$.

Notice that $\mathcal{T}_{2}$ is $(2,2)$-weighted but not 3 -weighted, since any new maximal segment intersects two of the other new maximal segments.

For a general hierarchical T-mesh, we consider a sequence of T-meshes $\mathcal{T}_{0}, \ldots, \mathcal{T}_{l}$ where $\mathcal{T}_{0}$ has one cell, $\mathcal{T}_{l}=\mathcal{T}$ and such that $\mathcal{T}_{i+1}$ refines $\mathcal{T}_{i}$ by inserting new edges. We can assume that at each level $i \neq 0$ a new maximal interior segment $\rho_{i}$ appears and that we number the maximal interior segments of $\mathcal{T}$ in the order they appear during this subdivision. Notice that any maximal interior segment of $\mathcal{T}$ extends one of the maximal interior segments $\rho_{i}$ and thus its weight is bigger. Notice also that the maximal interior segment $\rho_{i}$ introduced at level $i$ extends to a maximal segment of $\mathcal{T}$, which may intersect the boundary. In this case, it is not involved in the dimension upper bound. Then, we have the following corollary:

Proposition 5.3. Let $\mathcal{T}$ be a hierarchical T-mesh.

$$
9 f_{2}-6 f_{1}^{o}+4 f_{0}^{o} \leq \operatorname{dim} \mathcal{S}_{2,2}^{1,1}(\mathcal{T}) \leq 9 f_{2}-6 f_{1}^{o}+4 f_{0}^{o}+\sigma
$$


where $\sigma$ is the number of levels of the subdivision where a maximal interior segment with no-interior point is introduced.

Proof. Consider the new maximal interior segment $\rho_{i}$ of $\mathcal{T}_{i}$ appearing at level $i$. By construction, the end points of $\rho_{i}$ are not on maximal interior segments of bigger index. Thus $\omega\left(\rho_{i}\right) \geq 2$. If $\rho_{i}$ contains an interior vertex then $\omega\left(\rho_{i}\right) \geq 3$ and $\left(3-\omega\left(\rho_{i}\right)\right)_{+}=0$. Otherwise $\left(3-\omega\left(\rho_{i}\right)\right)_{+}=1$. As $\rho_{i}$ extends to a maximal segment $\tilde{\rho}_{i}$ which is either interior or intersecting the boundary, we have $\left(3-\omega\left(\tilde{\rho}_{i}\right)\right)_{+} \leq$ $\left(3-\omega\left(\rho_{i}\right)\right)_{+}$with the convention that $\omega\left(\tilde{\rho}_{i}\right)=3$ if $\tilde{\rho}_{i}$ is intersecting the boundary.

By Theorem 3.7, we have

$$
0 \leq h_{2,2}^{1,1}(\mathcal{T}) \leq \sum_{i=1}^{l}\left(3-\omega\left(\tilde{\rho}_{i}\right)\right)_{+} \leq \sum_{i=1}^{l}\left(3-\omega\left(\rho_{i}\right)\right)_{+}
$$

where $l$ is the number of levels in the subdivision, $\rho_{i}$ is the maximal interior segment introduced at level $i$ and $\tilde{\rho}_{i}$ is its extension in $\mathcal{T}$. By the previous remarks, $\sum_{i=1}^{l}(3-$ $\left.\omega\left(\rho_{i}\right)\right)_{+}=\sigma$ is the number of levels of the subdivision where a maximal interior segment with no interior point is introduced. Using Theorem 3.1, this proves the bound on the dimension of $\mathcal{S}_{2,2}^{1,1}(\mathcal{T})$.

Examples 5.1 and 5.2 show that the dimension can be given by the upper bound. On other other hand, for any (3,3)-weighted hierarchical subdivision, the lower bound is reached. This shows that the inequalities (5.3) are optimal for $\operatorname{dim} \mathcal{S}_{2,2}^{1,1}(\mathcal{T})$.

Remark 5.4. In the case of a hierarchical subdivision where some cells of a given level are subdivided (as $\sigma_{1}$ in Example 5.2) into 9 sub-cells which have the same length and height, it can be proved that the dimension is in fact,

$$
\operatorname{dim} \mathcal{S}_{2,2}^{1,1}(\mathcal{T})=9 f_{2}-6 f_{1}^{o}+4 f_{0}^{o}+\sigma,
$$

where $\sigma$ is the number of isolated subdivided cells (i.e. the cell is subdivided, not touching the boundary and the adjacent cells sharing an edge are not subdivided) at some level of the subdivision. Indeed, any maximal interior segment subdividing a non-isolated cell contains an interior point and is not involved in the upper bound. As in Example 5.2, only the isolated cells have a maximal interior segment with no interior points. This example also shows that a new $C^{(1,1)}$ bi-quadratic basis element can be constructed for each isolated cell, proving that the upper bound is reached. This gives a dimension formula similar to the one in [7, for a slightly different subdivision strategy.

5.3. Bicubic $C^{1,1}$ T-splines. For $m=m^{\prime}=3$ and $r=r^{\prime}=1$, that is, for piecewise bicubic polynomial functions which are $C^{1}$, Proposition 4.3 yields $h_{3,3}^{1,1}(\mathcal{T})=0$. Using Theorem 3.1 and Lemma A.1 in the appendix, we obtain:

$$
\operatorname{dim} \mathcal{S}_{3,3}^{2,2}(\mathcal{T})=16 f_{2}-8 f_{1}^{o}+4 f_{0}^{o}=4\left(f_{0}^{+}+f_{0}^{b}\right) .
$$

5.4. Bicubic $C^{2,2}$ T-splines. For $m=m^{\prime}=3$ and $r=r^{\prime}=2$, by Theorem 3.1 and Lemma A.1, we have:

$$
\operatorname{dim} \mathcal{S}_{3,3}^{2,2}(\mathcal{T})=16 f_{2}-12 f_{1}^{o}+9 f_{0}^{o}+h_{3,3}^{2,2}(\mathcal{T})=f_{0}^{+}-f_{0}^{T}+2 f_{0}^{b}+8+h_{3,3}^{2,2}(\mathcal{T})
$$

If $\mathcal{T}$ is a hierarchical $(4,4)$-weighted subdivision, then by Theorem 3.9, we have $h_{3,3}^{2,2}(\mathcal{T})=0$. 


\section{Appendix A. Combinatorial properties}

We recall some well-known enumeration results for a T-mesh of an axis-aligned rectangular domain $\Omega$.

\section{Lemma A.1.}

- $f_{2}=f_{0}^{+}+\frac{1}{2} f_{0}^{T}+\frac{1}{2} f_{0}^{b}-1$,

- $f_{1}^{o}=2 f_{0}^{+}+\frac{3}{2} f_{0}^{T}+\frac{1}{2} f_{0}^{b}-2$,

- $f_{0}^{o}=f_{0}^{+}+f_{0}^{T}$.

Proof. Each face $\sigma \in \mathcal{T}_{2}$ is a rectangle with 4 corners. If we count these corners for all cells in $\mathcal{T}_{2}$, we enumerate 4 times the crossing vertices, 2 times the $T$-vertices which are interior or on the boundary and one time the corner vertices of $\Omega$. This yields the relation

$$
4 f_{2}=4 f_{0}^{+}+2\left(f_{0}^{T}+\left(f_{0}^{b}-4\right)\right)+4 .
$$

Each interior edge $\tau \in \mathcal{T}_{1}^{o}$ has two end points. Counting these end points for all interior edges, we count 4 times the crossing vertices, 3 times the $T$-vertices which are interior and one time the $T$-vertices on the boundary:

$$
2 f_{1}^{o}=4 f_{0}^{+}+3 f_{0}^{T}+\left(f_{0}^{b}-4\right) .
$$

Finally, as an interior vertex is a crossing vertex or a $T$-vertex, we have

$$
f_{0}^{o}=f_{0}^{+}+f_{0}^{T} .
$$

\section{Appendix B. Complexes And homology}

Let us recall here the basic properties that we will need on complexes of vector spaces. Given a sequence of $\mathbb{K}$-vectors spaces $A_{i}, i=0, \ldots, l$ and linear maps $\partial_{i}: A_{i} \rightarrow A_{i+1}$, we say that we have a complex

$$
\mathcal{A}: A_{l} \rightarrow A_{l-1} \rightarrow \cdots A_{i} \rightarrow A_{i-1} \rightarrow \cdots A_{1} \rightarrow A_{0}
$$

if $\operatorname{im} \partial_{i} \subset \operatorname{ker} \partial_{i-1}$.

Definition B.1. The $i^{\text {th }}$ homology $H_{i}(\mathcal{A})$ of $\mathcal{A}$ is $\operatorname{ker} \partial_{i-1} / \operatorname{im} \partial_{i}$ for $i=1, \ldots, l$.

The complex $\mathcal{A}$ is called exact (or an exact sequence) if $H_{i}(\mathcal{A})=0$ (i.e. im $\partial_{i}=$ ker $\left.\partial_{i-1}\right)$ for $i=1, \ldots, l$. If the complex is exact and $A_{l}=A_{0}=0$, we have

$$
\sum_{i=1}^{l-1}(-1)^{i} \operatorname{dim} A_{i}=0 .
$$

Given complexes $\mathcal{A}=\left(A_{i}\right)_{i=0, \ldots, l} \mathcal{B}=\left(\mathcal{B}_{i}\right)_{i=0, \ldots, l}, \mathcal{C}=\left(C_{i}\right)_{i=0, \ldots, l}$ and exact sequences

$$
0 \rightarrow A_{i} \rightarrow B_{i} \rightarrow C_{i} \rightarrow 0
$$

for $i=0, \ldots, l$, we have a long exact sequence [4, [22, p. 182]:

$$
\cdots \rightarrow H_{i+1}(\mathcal{C}) \rightarrow H_{i}(\mathcal{A}) \rightarrow H_{i}(\mathcal{B}) \rightarrow H_{i}(\mathcal{C}) \rightarrow H_{i-1}(\mathcal{A}) \rightarrow \cdots
$$




\section{Appendix C. Dual topological complex}

The dual complex $\mathcal{T}^{\star}$ of the subdivision $\mathcal{T}$, is such that we have the following properties.

- a face $\sigma \in \mathcal{T}_{2}$ is a vertex of the dual complex $\mathcal{T}^{\star}$.

- an edge of $\mathcal{T}^{\star}$ is connecting two elements $\sigma, \sigma^{\prime} \in \mathcal{T}_{2}$ if they share a common (interior) edge $\tau \in \mathcal{T}_{1}^{o}$. Thus it is identified with the edge $\tau$ of $\mathcal{T}$ between $\sigma, \sigma^{\prime}$

- a face of $\mathcal{T}^{\star}$ corresponds to an elements $\gamma \in \mathcal{T}_{0}^{o}$. It is either a triangle if $\gamma$ is a $\mathrm{T}$-junction or a quadrangle if $\gamma$ is a crossing vertex.

Notice that the boundary cells of $\mathcal{T}$ correspond to boundary vertices of $\mathcal{T}^{\star}$. They are connected by boundary edges which belong to a single face of $\mathcal{T}^{\star}$.

\section{Appendix D. Topological Chain COMPleX}

In this appendix section, we recall the main properties of the topological chain complex

$$
\Re_{m, m^{\prime}}\left(\mathcal{T}^{o}\right): \bigoplus_{\sigma \in \mathcal{T}_{2}}[\sigma] R_{m, m^{\prime}} \rightarrow \bigoplus_{\tau \in \mathcal{T}_{1}^{o}}[\tau] R_{m, m^{\prime}} \rightarrow \bigoplus_{\gamma \in \mathcal{T}_{0}^{o}}[\gamma] R_{m, m^{\prime}} \rightarrow 0
$$

where

- $\forall \gamma \in \mathcal{T}_{o}^{o}, \partial_{0}([\gamma])=0$

- $\forall \tau=\left[\gamma_{1}, \gamma_{2}\right] \in \mathcal{T}_{1}^{o}, \partial_{1}\left(\left[\gamma_{1}, \gamma_{2}\right]\right)=\left[\gamma_{1}\right]-\left[\gamma_{2}\right]$ with $[\gamma] \equiv 0$ iff $\gamma \in \partial \Omega$, and

- $\forall \sigma \in \mathcal{T}_{2}^{o}$ with its counterclockwise boundary formed by the edges $\left[\gamma_{1}, \gamma_{2}\right]$, $\ldots,\left[\gamma_{l}, \gamma_{1}\right], \partial_{2}(\sigma)=\left[\gamma_{1}, \gamma_{2}\right]+\cdots+\left[\gamma_{l}, \gamma_{1}\right]$ with $\left[\gamma, \gamma^{\prime}\right] \equiv 0$ iff $\gamma, \gamma^{\prime} \in \partial \Omega$.

We assume that $\Omega$ is simply connected and $\Omega^{\circ}$ is connected. We prove that $\mathfrak{R}_{m, m^{\prime}}\left(\mathcal{T}^{o}\right)$ is acyclic on a T-mesh of $\Omega$.

Proposition D.1. $H_{0}\left(\Re_{m, m^{\prime}}\left(\mathcal{T}^{o}\right)\right)=0$.

Proof. Let $\gamma \in \mathcal{T}_{0}^{o}$. There is a sequence of edges $\tau_{0}=\gamma_{0} \gamma_{1}, \tau_{1}=\gamma_{1} \gamma_{2}, \ldots, \tau_{l}=$ $\gamma_{l} \gamma_{l+1}$, such that $\tau_{i} \in \mathcal{T}_{1}^{o}, \gamma_{0} \notin \mathcal{T}_{0}^{o}$ and $\gamma_{l+1}=\gamma$. Then

$$
\partial_{1}\left(\left[\tau_{0}\right]+\cdots+\left[\tau_{l}\right]\right)=\left[\gamma_{1}\right]-\left[\gamma_{0}\right]+\cdots+\left[\gamma_{l+1}\right]-\left[\gamma_{l}\right]=[\gamma]
$$

since $\left[\gamma_{0}\right]=0$ and $\left[\gamma_{l+1}\right]=[\gamma]$. Multiplying by any element in $R_{m, m^{\prime}}$, we get that $[\gamma] R_{m, m^{\prime}} \subset \operatorname{im} \partial_{1}$ and thus $H_{0}\left(\Re_{m, m^{\prime}}\left(\mathcal{T}^{o}\right)\right)=0$.

Proposition D.2. $H_{1}\left(\Re_{m, m^{\prime}}\left(\mathcal{T}^{o}\right)\right)=0$.

Proof. Let $p=\sum_{\tau \in \mathcal{T}_{1}^{\circ}}[\tau] p_{\tau} \in \operatorname{ker} \partial_{1}$ with $p_{\tau} \in R_{m, m^{\prime}}$. Let us prove that $p$ is in the image of $\partial_{2}$. For each $\gamma \in \mathcal{T}_{0}^{o}$ and each edge $\tau$ which contains $\gamma$, we have $\sum \varepsilon_{\tau} p_{\tau}=0$ with $\varepsilon_{\tau}=1$ if $\tau$ ends at $\gamma, \varepsilon_{\tau}=-1$ if $\tau$ starts at $\gamma$ and $\varepsilon_{\tau}=0$ otherwise.

For any $\sigma \in \mathcal{T}_{2}^{o}$ and $\tau \in \mathcal{T}_{1}^{o}$, we define $\varepsilon_{\sigma, \tau}=1$ if $\tau$ is oriented counterclockwise on the boundary of $\sigma, \varepsilon_{\sigma, \tau}=-1$ if $\tau$ is oriented counterclockwise on the boundary of $\sigma$ and $\varepsilon_{\sigma, \tau}=0$ otherwise.

For any oriented edge of the dual graph $\mathcal{T}^{\star}$ from $\sigma^{\prime}$ to $\sigma$, let us define $\partial_{1}^{\star}\left(\left[\sigma^{\prime}, \sigma\right]\right)=$ $\varepsilon_{\sigma, \tau} p_{\tau}$. Notice that $\partial_{1}^{\star}\left(\left[\sigma, \sigma^{\prime}\right]\right)=\varepsilon_{\sigma^{\prime}, \tau} p_{\tau}=-\varepsilon_{\sigma, \tau} p_{\tau}=-\partial_{1}^{\star}\left(\left[\sigma^{\prime}, \sigma\right]\right)$, since the orientation of $\tau$ on the boundary of $\sigma$ and $\sigma^{\prime}$ are opposite.

Let $\sigma_{0}$ be the 2-face of $\mathcal{T}$ with the lowest left corner for the lexicographic ordering. We order the cells $\sigma \in \mathcal{T}_{2}$ according to their distance to $\sigma_{0}$ in this dual graph $\mathcal{T}^{\star}$. 
We define an element $q=\sum_{\sigma \in \mathcal{T}_{2}} q_{\sigma}[\sigma]$ where $q_{\sigma} \in R_{m, m^{\prime}}$ by induction using this order, as follows:

- $q_{\sigma_{0}}=0$;

- For any $\sigma, \sigma^{\prime} \in \mathcal{T}_{2}$, if $\sigma>\sigma^{\prime}$ and $\sigma$ and $\sigma^{\prime}$ share a common edge $\tau$, then $q_{\sigma}=q_{\sigma^{\prime}}+\partial_{1}^{\star}\left(\left[\sigma^{\prime}, \sigma\right]\right)$.

Thus, if $\left[\sigma_{0}, \sigma_{1}\right],\left[\sigma_{1}, \sigma_{2}\right], \ldots,\left[\sigma_{k-1}, \sigma_{k}\right]$ is a path of $\mathcal{T}^{\star}$ connecting $\sigma_{0}$ to $\sigma_{k}=\sigma$ with $\sigma_{i+1}>\sigma_{i}$ then $q_{\sigma}=\sum_{i=0}^{k-1} \partial_{1}^{\star}\left(\left[\sigma_{i}, \sigma_{i+1}\right]\right)$. Let us prove that this definition does not depend on the chosen path between $\sigma_{0}$ and $\sigma$.

We first show that for any face $\gamma^{\star}$ of $\mathcal{T}^{\star}$ attached to a vertex $\gamma$, if its counterclockwise boundary is formed by the edges $\left[\sigma, \sigma^{\prime}\right],\left[\sigma^{\prime}, \sigma^{\prime \prime}\right], \ldots,\left[\sigma^{\prime \prime \prime}, \sigma\right]$ corresponding to the edges $\tau, \tau^{\prime}, \tau^{\prime \prime}, \ldots$ of $\mathcal{T}$ containing $\gamma$, then

$\partial_{1}^{\star}\left(\left[\sigma, \sigma^{\prime}\right]\right)+\partial_{1}^{\star}\left(\left[\sigma^{\prime}, \sigma^{\prime \prime}\right]+\cdots+\partial_{1}^{\star}\left(\left[\sigma^{\prime \prime \prime}, \sigma\right]\right)=\varepsilon_{\sigma, \tau} p_{\tau}+\varepsilon_{\sigma^{\prime}, \tau^{\prime}} p_{\tau^{\prime}}+\varepsilon_{\sigma^{\prime \prime}, \tau^{\prime \prime}} p_{\tau^{\prime \prime}}+\cdots=0\right.$.

By changing the orientation of an edge $\tau$, we replace $p_{\tau}$ by $-p_{\tau}$ and $\varepsilon_{\sigma, \tau}$ by $-\varepsilon_{\sigma, \tau}$ so that the quantity $\varepsilon_{\sigma, \tau} p_{\tau}$ is not changed. Thus we can assume that all the edges $\tau, \tau^{\prime}, \tau^{\prime \prime}, \ldots$ are pointing to $\gamma$. As $p \in \operatorname{ker} \partial_{1}$, we have $p_{\tau}+p_{\tau^{\prime}}+p_{\tau^{\prime \prime}}+\cdots=0$.

Now as the cells $\sigma, \sigma^{\prime}, \sigma^{\prime \prime}, \ldots, \sigma$ are ordered counterclockwise around $\gamma$ and as the edges are pointing to $\gamma$, we have $\varepsilon_{\sigma, \tau}=\varepsilon_{\sigma^{\prime}, \tau^{\prime}}=\varepsilon_{\sigma^{\prime \prime}, \tau^{\prime \prime}}=\cdots=1$, so that the sum $\partial_{1}^{\star}\left(\left[\sigma, \sigma^{\prime}\right]\right)+\partial_{1}^{\star}\left(\left[\sigma^{\prime}, \sigma^{\prime \prime}\right]+\cdots+\partial_{1}^{\star}\left(\left[\sigma^{\prime \prime \prime}, \sigma\right]\right)\right.$ over the boundary of a face $\gamma^{\star}$ of $\mathcal{T}^{\star}$ is 0 .

By composition, for any loop of $\mathcal{T}^{\star}$, the sum on the corresponding oriented edges is 0 . This shows that the definition of $q_{\sigma}$ does not depend on the oriented path from $\sigma_{0}$ to $\sigma$.

By construction, we have

$$
\partial_{2}(q)=\sum_{\sigma \in \mathcal{T}_{2}}\left(\sum_{\tau \in \mathcal{T}_{1}^{o}} \varepsilon_{\sigma, \tau} q_{\sigma}[\tau]\right)=\sum_{\tau \in \mathcal{T}_{1}^{o}}\left(\sum_{\sigma \in \mathcal{T}_{2}} \varepsilon_{\sigma, \tau} q_{\sigma}\right)[\tau]
$$

For each interior edge $\tau \in \mathcal{T}_{1}^{o}$, there are two faces $\sigma_{1}>\sigma_{2}$, which are adjacent to $\tau$. Thus, we have $\varepsilon_{\sigma_{1}, \tau}=-\varepsilon_{\sigma_{2}, \tau}$ and $q_{\sigma_{1}}=q_{\sigma_{2}}+\varepsilon_{\sigma_{1}, \tau} p_{\tau}$. We deduce that

$$
\left(\sum_{\sigma} \varepsilon_{\sigma, \tau} q_{\sigma}\right)=\varepsilon_{\sigma_{1}, \tau} q_{\sigma_{1}}+\varepsilon_{\sigma_{2}, \tau} q_{\sigma_{2}}=\varepsilon_{\sigma_{1}, \tau}\left(q_{\sigma_{2}}+\varepsilon_{\sigma_{1}, \tau} p_{\tau}\right)+\varepsilon_{\sigma_{2}, \tau} q_{\sigma_{2}}=p_{\tau} .
$$

This shows that $\partial_{2}(q)=p$. In other words, $\operatorname{im} \partial_{2}=\operatorname{ker} \partial_{1}$ and $H_{1}\left(\Re_{m, m^{\prime}}\left(\mathcal{T}^{o}\right)\right)=$ 0 .

Proposition D.3. If $\Omega$ has one connected component. Then $H_{2}\left(\Re_{m, m^{\prime}}\left(\mathcal{T}^{o}\right)\right)=$ $R_{m, m^{\prime}}$.

Proof. An element of $H_{2}\left(\Re_{m, m^{\prime}}\left(\mathcal{T}^{o}\right)\right)=\operatorname{ker} \partial_{2}$ is a collection of polynomials $\left(p_{\sigma}\right)_{\sigma \in \mathcal{T}_{2}}$ such that $p_{\sigma} \in R_{m, m^{\prime}}$ and $p_{\sigma}=p_{\sigma^{\prime}}$ if $\sigma$ and $\sigma^{\prime}$ share an (internal) edge. As $\mathcal{T}$ is a subdivision of a rectangle $D_{0}$, all faces $\sigma \in \mathcal{T}_{2}$ share pairwise an edge. Thus $p_{\sigma}=p_{\sigma^{\prime}}$ for all $\sigma, \sigma^{\prime} \in \mathcal{T}_{2}$ and $H_{2}\left(\Re_{m, m^{\prime}}(\mathcal{T})\right)=R_{m, m^{\prime}}$.

Notice that by counting the dimensions in the exact sequence $\Re_{m, m^{\prime}}(\mathcal{T})$, we recover the well-known Euler formula: $f_{2}-f_{1}+f_{0}=1$ (the domain $\Omega$ has one connected component). 


\section{REFERENCES}

[1] Dmitry Berdinsky, Min-jae Oh, Tae-wan Kim, and Bernard Mourrain. On the problem of instability in the dimension of a spline space over a T-mesh. Comput. Graph., 36(5):507-513, 2012.

[2] Louis J. Billera, Homology of smooth splines: generic triangulations and a conjecture of Strang, Trans. Amer. Math. Soc. 310 (1988), no. 1, 325-340, DOI 10.2307/2001125. MR.965757(89k:41010)

[3] A. Buffa, D. Cho, and G. Sangalli, Linear independence of the T-spline blending functions associated with some particular T-meshes, Comput. Methods Appl. Mech. Engrg. 199 (2010), no. 23-24, 1437-1445, DOI 10.1016/j.cma.2009.12.004. MR2630153(2011d:65042)

[4] Henri Cartan and Samuel Eilenberg, Homological algebra, Princeton Landmarks in Mathematics, Princeton University Press, Princeton, NJ, 1999. With an appendix by David A. Buchsbaum; Reprint of the 1956 original. MR.1731415 (2000h:18022)

[5] Carl de Boor, A practical guide to splines, Revised edition, Applied Mathematical Sciences, vol. 27, Springer-Verlag, New York, 2001. MR1900298 (2003f:41001)

[6] Jiansong Deng, Falai Chen, and Yuyu Feng, Dimensions of spline spaces over T-meshes, J. Comput. Appl. Math. 194 (2006), no. 2, 267-283, DOI 10.1016/j.cam.2005.07.009. MR2239393(2007a:41017)

[7] Jiansong Deng, Falai Chen, and Liangbing Jin, Dimensions of biquadratic spline spaces over T-meshes, J. Comput. Appl. Math. 238 (2013), 68-94, DOI 10.1016/j.cam.2012.08.020. MR2972590

[8] Jiansong Deng, Falai Chen, Xin Li, Changqi Hu, Weihua Tong, Zhouwang Yang, and Yuyu Feng. Polynomial splines over hierarchical T-meshes. Graph. Models, 70(4):76-86, 2008.

[9] Tor Dokken, Tom Lyche, and Kjell-Fredrik Pettersen. Polynomial splines over locally refined box-partitions, Comput. Aided Geom. Design, 30(3):331-356, 2013. MR.3019748

[10] Richard Ehrenborg and Gian-Carlo Rota, Apolarity and canonical forms for homogeneous polynomials, European J. Combin. 14 (1993), no. 3, 157-181, DOI 10.1006/eujc.1993.1022. MR 1215329 (94e:15062)

[11] Gerald Farin. Curves and Surfaces for Computer Aided Geometric Design: A Practical Guide, 5th Edition. Morgan Kaufmann, San Mateo, CA, 2001.

[12] Allen Hatcher, Algebraic topology, Cambridge University Press, Cambridge, 2002. MR.1867354(2002k:55001)

[13] Zhang-jin Huang, Jian-song Deng, Yu-yu Feng, and Fa-lai Chen, New proof of dimension formula of spline spaces over T-meshes via smoothing cofactors, J. Comput. Math. 24 (2006), no. 4, 501-514. MR2243118(2007a:41019)

[14] T. J. R. Hughes, J. A. Cottrell, and Y. Bazilevs, Isogeometric analysis: CAD, finite elements, NURBS, exact geometry and mesh refinement, Comput. Methods Appl. Mech. Engrg. 194 (2005), no. 39-41, 4135-4195, DOI 10.1016/j.cma.2004.10.008. MR2152382(2006a:65018)

[15] Joseph P. S. Kung and Gian-Carlo Rota, The invariant theory of binary forms, Bull. Amer. Math. Soc. (N.S.) 10 (1984), no. 1, 27-85, DOI 10.1090/S0273-0979-1984-15188-7. MR722856 (85g:05002)

[16] Chong-Jun Li, Ren-Hong Wang, and Feng Zhang. Improvement on the dimensions of spline spaces on T-mesh. Journal of Information $\&$ Computational Science, 3(2):235-244, 2006.

[17] Xin Li and Falai Chen, On the instability in the dimension of splines spaces over T-meshes, Comput. Aided Geom. Design 28 (2011), no. 7, 420-426, DOI 10.1016/j.cagd.2011.08.001. MR2836487 (2012m:65035)

[18] G. Salmon. Modern Higher Algebra. G.E. Stechert and Co., New York, 1885. Reprinted 1924.

[19] Hal Schenck and Mike Stillman, Local cohomology of bivariate splines, J. Pure Appl. Algebra 117/118 (1997), 535-548, DOI 10.1016/S0022-4049(97)00026-1. Algorithms for algebra (Eindhoven, 1996). MR1457854(99d:13011)

[20] Thomas W. Sederberg, David L. Cardon, G. Thomas Finnigan, Nicholas S. North, Jianmin Zheng, and Tom Lyche. T-spline simplification and local refinement. ACM Trans. Graph., 23(3):276-283, 2004.

[21] Thomas W. Sederberg, Jianmin Zheng, Almaz Bakenov, and Ahmad Nasri. T-splines and T-nurccs. ACM Trans. Graph., 22(3):477-484, 2003.

[22] Edwin H. Spanier, Algebraic topology, McGraw-Hill Book Co., New York, 1966. MR.0210112 (35 \#1007) 
[23] Ren-Hong Wang, Multivariate spline functions and their applications, Mathematics and its Applications, vol. 529, Kluwer Academic Publishers, Dordrecht, 2001. Translated from the 1994 Chinese original by Shao-Ming Wang and revised by the author. MR.1891792 (2003f:41002)

Galaad, Inria Méditerranée, 2004 route des Lucioles, BP 93, 06902 Sophia Antipolis, FRANCE

E-mail address: Bernard.Mourrain@inria.fr 